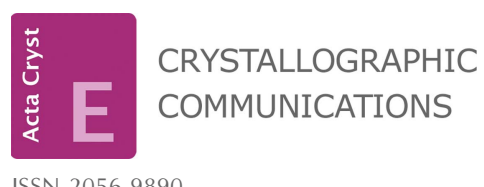

ISSN 2056-9890
Received 20 February 2019

Accepted 26 February 2019

Edited by M. Weil, Vienna University of

Technology, Austria

Keywords: crystal structure; binuclear; lanthanide; oxalate bridge; $N, N^{\prime}$-bis(2-hydroxybenzyl)-N, $N^{\prime}$-bis(pyridin-2-ylmethyl)ethylenediamine; $\mathrm{H}_{2}$ bbpen.

CCDC references: 1899963; 1899964

Supporting information: this article has supporting information at journals.iucr.org/e

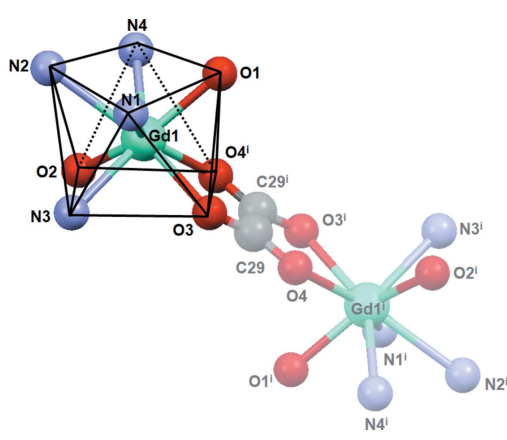

OPEN $\odot$ ACCESS

\section{Crystal structures of binuclear complexes of gadolinium(III) and dysprosium(III) with oxalate bridges and chelating $N, N^{\prime}$-bis(2-oxidobenzyl)- $N, N^{\prime}$-bis(pyridin-2-ylmethyl)ethylenediamine (bbpen $^{2-}$ )}

\author{
Guilherme Augusto Barbosa, Francielli Sousa Santana, Giovana Gioppo Nunes and \\ Jaísa Fernandes Soares*
}

Departamento de Química, Universidade Federal do Paraná, Centro, Politécnico, Jardim das Américas, 81530-900,
Curitiba-PR, Brazil. *Correspondence e-mail: jaisa@quimica.ufpr.br

The reaction between mononuclear $[\operatorname{Ln}($ bbpen $) \mathrm{Cl}]\left[\operatorname{Ln}=\mathrm{Gd}\right.$ or Dy; $\mathrm{H}_{2}$ bbpen $=$ $N, N^{\prime}$-bis(2-hydroxybenzyl)- $N, N^{\prime}$-bis(pyridin-2-ylmethyl)ethylenediamine, $\mathrm{C}_{28} \mathrm{H}_{30} \mathrm{~N}_{4} \mathrm{O}_{2}$ ] and potassium oxalate monohydrate in water/methanol produced the solvated centrosymmetric isostructural binuclear ( $\mu$-oxalato)bis $\left\{\left[N, N^{\prime}\right.\right.$ bis(2-oxidobenzyl- $\kappa O$ )- $N, N^{\prime}$-bis(pyridin-2-ylmethyl- $\kappa N$ )ethylenediamine$\left.\kappa^{2} N, N^{\prime}\right]$ dilanthanide(III) $\}$-methanol-water $(1 / 4 / 4)$ complexes, $\left[\operatorname{Ln}_{2}\left(\mathrm{C}_{28} \mathrm{H}_{28^{-}}\right.\right.$ $\left.\left.\mathrm{N}_{4} \mathrm{O}_{2}\right)_{2}\left(\mathrm{C}_{2} \mathrm{O}_{4}\right)\right] \cdot 4 \mathrm{CH}_{3} \mathrm{OH} \cdot 4 \mathrm{H}_{2} \mathrm{O}$, with lanthanide(III) $=$ gadolinium $(\mathrm{III})(\mathrm{Ln}=$ $\mathrm{Gd})$ and dysprosium(III) ( $L n=\mathrm{Dy})$, in high yields ( $c a 70 \%)$ directly from the reaction mixtures. In both complexes, the lanthanide ion is eight-coordinate and adopts a distorted square-antiprismatic coordination environment. The triclinic $(P \overline{1})$ unit cell contains one dimeric unit together with four water and four methanol molecules; in the final structural model, two of each type of solvating molecule refine well. In each lanthanide(III) dimeric molecule, the mediumstrength $\mathrm{O} \cdots \mathrm{H}-\mathrm{O}$ hydrogen-bonding pattern involves four oxygen atoms, two of them from the phenolate groups that are 'bridged' by one water and one methanol molecule. These interactions seem to contribute to the stabilization of the relatively compact shape of the dimer. Electron densities associated with an additional water and methanol molecule were removed with the SQUEEZE procedure in PLATON [Spek (2015). Acta Cryst. C71, 9-18]. These two new compounds are of interest with respect to magnetic properties.

\section{Chemical context}

Since the discovery, in 2003, of the first lanthanide(III)-based single-ion magnets $(\mathrm{SIM})$, namely $\left(\mathrm{Bu}_{4} \mathrm{~N}\right)\left[\operatorname{Ln} \mathrm{Pc}_{2}\right]\left(\mathrm{H}_{2} \mathrm{Pc}=\right.$ phthalocyanine; $L n=\mathrm{Tb}$ and Dy; Ishikawa et al., 2003), a number of lanthanide(III) complexes have been prepared for magnetic studies because of their intrinsically high magnetic anisotropy barrier. Heterometallic $3 d-4 f$ single-molecule magnets (SMM) have also been sought, particularly in the early 2000s, mainly because of the possibility of improving magnetic response when compared to $d$-block-only metal complexes such as those of manganese(III), cobalt(II) and nickel(II) (Piquer \& Sañudo, 2015).

Among the $3 d-4 f$ heterometallic systems of higher nuclearity, two tetranuclear compounds formulated as $[M(\mu-$ dto $\left.)_{3}\left\{\mathrm{Dy}\left(\mathrm{HBpz}_{3}\right)_{2}\right\}_{3}\right] \cdot 4 \mathrm{CH}_{3} \mathrm{CN} \cdot 2 \mathrm{CH}_{2} \mathrm{Cl}_{2}\left(M=\mathrm{Fe}^{\mathrm{III}}\right.$ or $\mathrm{Co}^{\mathrm{III}}$; $\mathrm{HBpz}^{-}=$hydrotris(pyrazolyl)borate; $\mathrm{dto}^{2-}=$ dithiooxalate) presented slow relaxation of the magnetization under applied magnetic field (Xu et al., 2012). In this three-blade propeller 
framework, the tris-chelate $\left[M(\mathrm{dto})_{3}\right]^{3-}$ complex forms the central unit, which is bridged to the $\left[\mathrm{Dy}\left(\mathrm{HBpz}_{3}\right)_{2}\right]^{+}$peripheral positions by the dithiooxalate ions. The lanthanide cations assume square-antiprismatic coordination environments while the $d$-block metal is octahedrally coordinated (Xu et al., 2012). The same monocationic $\left[\mathrm{Dy}\left(\mathrm{HBpz}_{3}\right)_{2}\right]^{+}$complex had previously been employed to produce binuclear $\left[\mathrm{Dy}_{2}(\mu\right.$-ox $)$ $\left.\left(\mathrm{HBpz}_{3}\right)_{4}\right] \cdot 2 \mathrm{CH}_{3} \mathrm{CN} \cdot \mathrm{CH}_{2} \mathrm{Cl}_{2}$, this time with oxalate $\left(\mathrm{ox}^{2-}\right)$ as the bridging ligand. Direct current (DC) magnetic susceptibility measurements performed with this dimeric compound revealed the presence of an intramolecular ferromagnetic interaction between the Dy ${ }^{\mathrm{III}}$ cations (Xu et al., 2010). Other oxalate-bridged lanthanide(III) complexes have also shown field-induced slow magnetic relaxation (Zhang et al., 2015) or weak (antiferro)magnetic exchange interactions (Feng et al., 2014). In all cases mentioned above, the products were obtained by self-assembly in one-pot reactions, sometimes under hydrothermal conditions.

In our research group, we first attempted to prepare heterometallic complexes of general formula $\left[M^{\mathrm{III}}(\mu-\mathrm{ox})_{3^{-}}\right.$ $\left.\{\operatorname{Ln}(\text { bbpen })\}_{3}\right]\left(\mathrm{H}_{2}\right.$ bbpen $=N, N^{\prime}$-bis $(2$-hydroxybenzyl $)-N, N^{\prime}$ bis(pyridin-2-ylmethyl)ethylenediamine) via modular synthesis employing [ $\operatorname{Ln}($ bbpen $) \mathrm{Cl}]\left(L n^{\mathrm{III}}=\mathrm{Gd}\right.$ or Dy) and $\mathrm{K}_{3}\left[M(\mathrm{ox})_{3}\right]\left(M^{\mathrm{III}}=\mathrm{Cr}\right.$ or $\left.\mathrm{Co}\right)$ as building blocks in a $3: 1$ proportion. The syntheses with gadolinium(III) and chromium(III) produced colourless crystals of the binuclear complex $\left[\{\mathrm{Gd}(\text { bbpen })\}_{2}(\mu\right.$-ox $\left.)\right] \cdot 4 \mathrm{CH}_{3} \mathrm{OH} \cdot 4 \mathrm{H}_{2} \mathrm{O}$, as revealed by single crystal X-ray diffraction analysis. The formation of this dimer is explained by dissociation of $\left[\mathrm{Cr}(\mathrm{ox})_{3}\right]^{3-}$ into $\left\{\mathrm{Cr}(\mathrm{ox})_{2}\left(\mathrm{OH}_{2}\right)_{2}\right\}^{-}$and $\mathrm{ox}^{2-}$ in aqueous solution (Krishnamurty \& Harris, 1960), followed by interaction of the $\mathrm{ox}^{2-}$ anion with $\mathrm{Gd}(\text { bbpen })^{+}$. Structural elucidation of this otherwise unexpected product prompted us to try and perform its targeted preparation with both gadolinium(III) and dysprosium(III) in good yields.

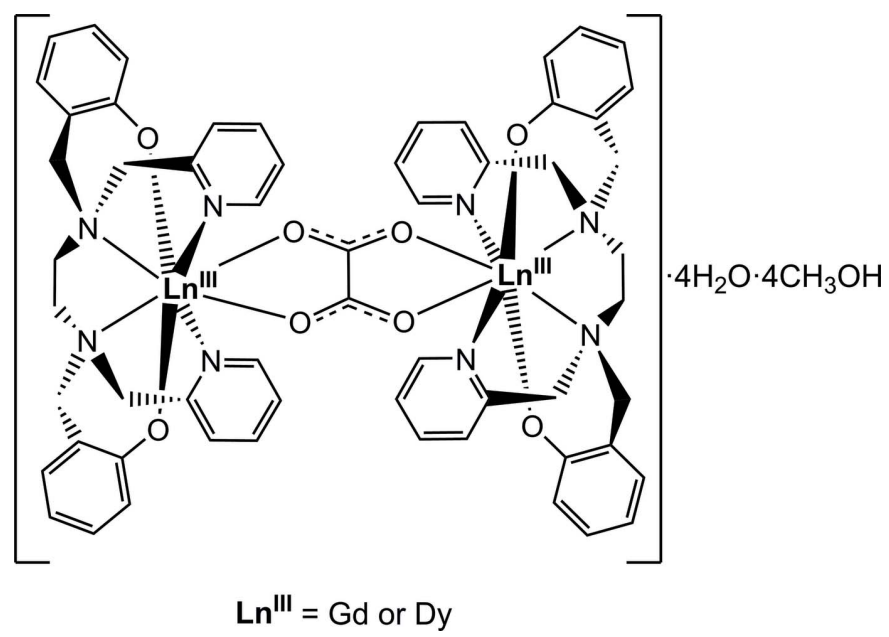

In this paper we report the rational synthesis and the crystal and molecular structures of the two binuclear and solvated $[\{L n \text { (bbpen })\}_{2}(\mu$-ox $\left.)\right]$ products $[L n=\mathrm{Gd}(\mathbf{1})$ or Dy (2) $]$, prepared from the direct reaction between $[\operatorname{Ln}($ bbpen $) \mathrm{Cl}]$ and $\mathrm{K}_{2} \mathrm{C}_{2} \mathrm{O}_{4} \cdot \mathrm{H}_{2} \mathrm{O}$ in water/methanol media.

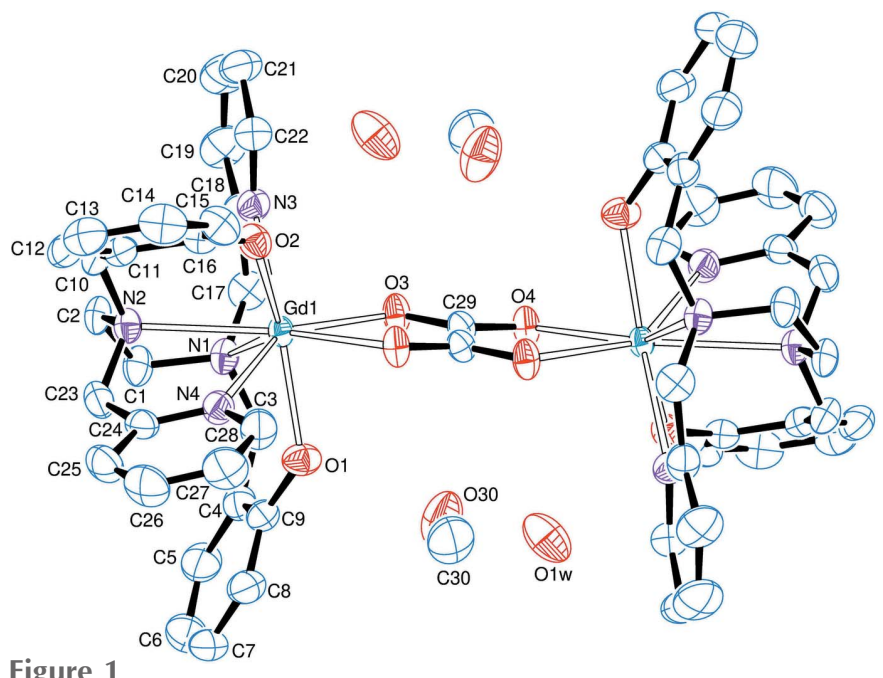

View of $\left[\{\mathrm{Gd}(\text { bbpen })\}_{2}(\mu\right.$-ox) $] \cdot 4 \mathrm{CH}_{3} \mathrm{OH} \cdot 4 \mathrm{H}_{2} \mathrm{O}$ (compound 1), with the atom-numbering scheme. Hydrogen atoms have been omitted for clarity. Displacement ellipsoids are drawn at the $50 \%$ probability level Unlabelled atoms are generated by the symmetry operation $-x,-y+1$, $-z+1$.

\section{Structural commentary}

Compounds $\mathbf{1}$ and $\mathbf{2}$ are isostructural and crystallize in the $P \overline{1}$ space group, with four methanol and four water molecules per lanthanide dimer. Crystals contain the neutral $\left[\operatorname{Ln}_{2}(\mu\right.$-ox $)$ (bbpen) $)_{2}$ ] molecules (Fig. 1) in which gadolinium(III) (1) or dysprosium(III) (2) are eight-coordinate; the $[\operatorname{Ln}(\text { bbpen })]^{+}$ units are connected to one another by oxalate bridging in the usual bis(bidentate) coordination mode. The $\mathrm{ox}^{2-}$ ligand lies about an inversion centre. The coordination sphere of the lanthanide(III) ion is formed by an $\mathrm{N}_{4} \mathrm{O}_{2}$ donor set from the bbpen $^{2-}$ ligand and two oxygen atoms from the bridging oxalate. In $\mathbf{1}$ and $\mathbf{2}$ each metal cation has a distorted squareantiprismatic coordination environment (Fig. 2), as indicated

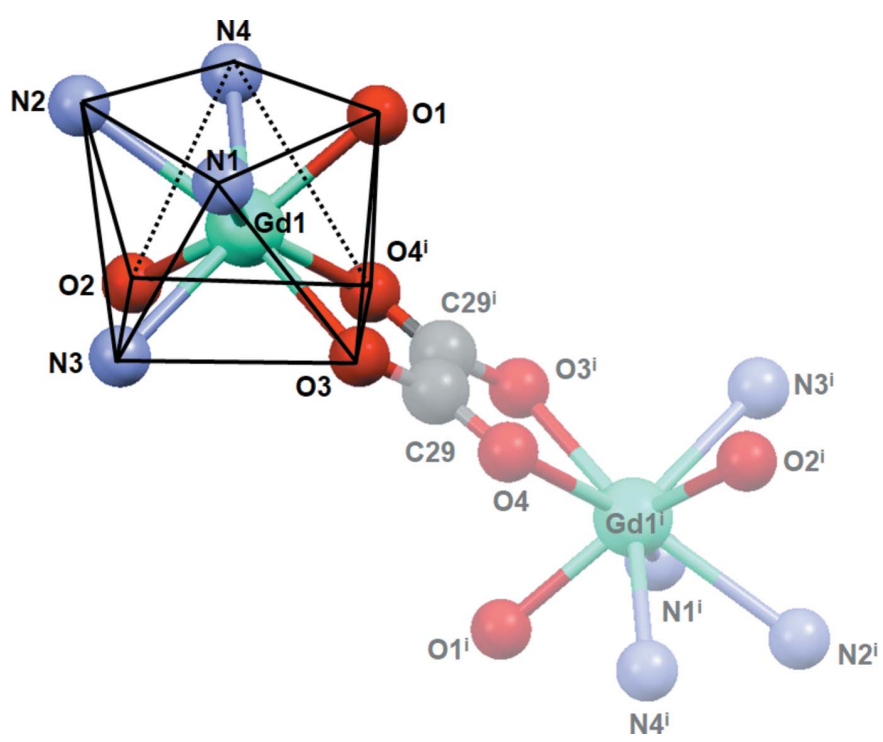

Figure 2

Plot of the coordination sphere about the lanthanide(III) atom in the structure of $\mathbf{1}$ [symmetry code: (i) $-x,-y+1,-z+1$ ]. 
Table 1

Hydrogen-bond geometry $\left(\AA,^{\circ}\right)$ for $\mathbf{1}$.

\begin{tabular}{lllll}
\hline$D-\mathrm{H} \cdots A$ & $D-\mathrm{H}$ & $\mathrm{H} \cdots A$ & $D \cdots A$ & $D-\mathrm{H} \cdots A$ \\
\hline $\mathrm{O} 30-\mathrm{H} 30 \cdots \mathrm{O} 1$ & 0.84 & 1.80 & $2.643(3)$ & 177 \\
$\mathrm{C} 1-\mathrm{H} 1 B \cdots \mathrm{O} 1 W^{\mathrm{ii}}$ & 0.99 & 2.59 & $3.459(3)$ & 147 \\
$\mathrm{O} 1 W-\mathrm{H} 1 W \cdots \mathrm{O} 2^{\mathrm{iii}}$ & $0.83(2)$ & $1.96(2)$ & $2.786(3)$ & $170(3)$ \\
$\mathrm{O} 1 W-\mathrm{H} 2 W \cdots \mathrm{O} 30$ & $0.88(2)$ & $1.87(2)$ & $2.745(3)$ & $171(4)$ \\
\hline
\end{tabular}

Symmetry codes: (ii) $-x+1,-y+1,-z+1$; (iii) $-x,-y+1,-z+1$.

by general inspection of atom positions and bond angles, and confirmed from the crystallographic data by the use of the $S H A P E$ program (Llunell et al., 2005). The average $L n-\mathrm{N}$ bonds are ca 2.60 and $2.58 \AA$ for $\mathbf{1}$ and $\mathbf{2}$, respectively, while the average $L n-\mathrm{O}$ distances are $c a 2.27$ (1) and $2.24 \AA$ (2). The non-bonding Dy...Dy distance in 2, 6.1488 (17) $\AA$, is close to the analogous distance of $6.14 \AA$ in $\left[\mathrm{Dy}_{2}(\mu\right.$-ox)$\left.\left(\mathrm{HBpz}_{3}\right)_{4}\right] \cdot 2 \mathrm{CH}_{3} \mathrm{CN} \cdot \mathrm{CH}_{2} \mathrm{Cl}_{2}$ (Xu et al., 2010). The O3-Ln$\mathrm{O} 4^{\mathrm{i}}$ angles of approximately $68^{\circ}$ in both $\mathbf{1}$ and $\mathbf{2}$ [symmetry code: (i) $-x, 1-y, 1-z$ ] are also similar to those reported for the dysprosium(III)-hydrotris(pirazolylborate) dimer mentioned above. The slightly decreased crystal volume of the Dy compound $\left[1626.3(7) \AA^{3}\right]$ compared with that of the Gd compound $\left[1633.7(3) \AA^{3}\right]$ is a perfect match with the smaller effective ionic radius of eight-coordinate $\mathrm{Dy}^{\mathrm{III}}$ versus $\mathrm{Gd}^{\mathrm{III}}$ (1.027 and $1.053 \AA$ A, respectively; Shannon, 1976), and is in line with the lanthanide contraction. Structural representations provided in this paper are for compound 1; the dysprosiu$\mathrm{m}$ (III) product $\mathbf{2}$ gives rise to very similar results.

\section{Supramolecular features}

In both structures, the hydrogen atoms from the crystallizing solvents (water and methanol) participate in an extensive

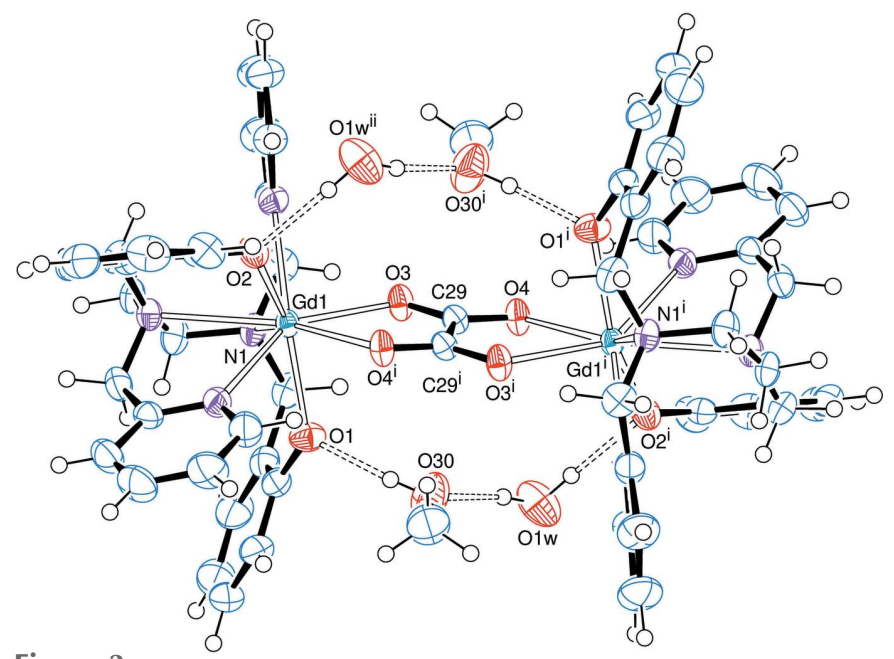

Figure 3

ORTEP representation of hydrogen-bonding interactions for compound 1 involving solvating methanol and water molecules, with hydrogen bonds indicated by double-dashed lines. Displacement ellipsoid are drawn at the $50 \%$ probability level [symmetry codes: (i) $-x,-y+1,-z+1$; (ii) $-x+1$, $-y+1,-z+1$.].
Table 2

Hydrogen-bond geometry $\left(\AA,^{\circ}\right)$ for 2 .

\begin{tabular}{lllll}
\hline$D-\mathrm{H} \cdots A$ & $D-\mathrm{H}$ & $\mathrm{H} \cdots A$ & $D \cdots A$ & $D-\mathrm{H} \cdots A$ \\
\hline $\mathrm{O} 30-\mathrm{H} 30 \cdots \mathrm{O} 1$ & 0.84 & 1.80 & $2.636(4)$ & 178 \\
$\mathrm{C} 1-\mathrm{H} 1 B \cdots \mathrm{O} 1 W^{\mathrm{ii}}$ & 0.99 & 2.58 & $3.448(4)$ & 146 \\
$\mathrm{O} 1 W-\mathrm{H} 1 W \cdots \mathrm{O} 2^{\mathrm{iii}}$ & $0.82(2)$ & $1.97(2)$ & $2.785(4)$ & $167(5)$ \\
$\mathrm{O} 1 W-\mathrm{H} 2 W \cdots \mathrm{O} 30$ & $0.86(2)$ & $1.95(3)$ & $2.759(5)$ & $158(5)$ \\
\hline
\end{tabular}

Symmetry codes: (ii) $-x+1,-y+1,-z+1$; (iii) $-x+2,-y+1,-z+1$.

three-dimensional hydrogen-bonding network that may be described as medium-strength intermolecular interactions (Tables 1 and 2).

The solvating (methanol and water) molecules, half of which refine well and are depicted in Fig. 3, participate in intermolecular interactions with the dimeric complexes $\mathbf{1}$ and 2. As seen in Fig. 3, one water and one methanol molecule are hydrogen-bonded to one another and to the phenolate oxygen atoms in the ligands, generating an $\mathrm{O} 1 \cdots \mathrm{H}-\mathrm{O} 30 \cdots \mathrm{H}-$ $\mathrm{O} 1 W-\mathrm{H} \cdots \mathrm{O} 2{ }^{\mathrm{iii}}$ 'bridge', as well as a symmetry-related chain on both sides of the plane formed by the metal and oxalate ions. The water molecules in these chains also connect one dimer to another through weak $\mathrm{C} 1-\mathrm{H} 1 B \cdots \mathrm{O} 1 W^{\mathrm{ii}}$ interactions (Fig. 4; Tables 1 and 2).

The other half of the solvent molecules in the unit cell, the electron densities of which have been removed with the SQUEEZE routine in PLATON (Spek, 2015) because of being highly disordered, also contribute to the overall hydrogen-bonding network. This is inferred from the positions of the four main electron-density peaks, which have been assigned to oxygen atoms from the disordered solvents and may give rise to medium-strength to weak hydrogen-bond interactions. For 1, O $\cdots \mathrm{O}$ distances involving three of these peaks amount to $2.66-2.78 \AA$ as far as $\mathrm{O} \cdots \mathrm{O} 1 \mathrm{~W}$ contacts are

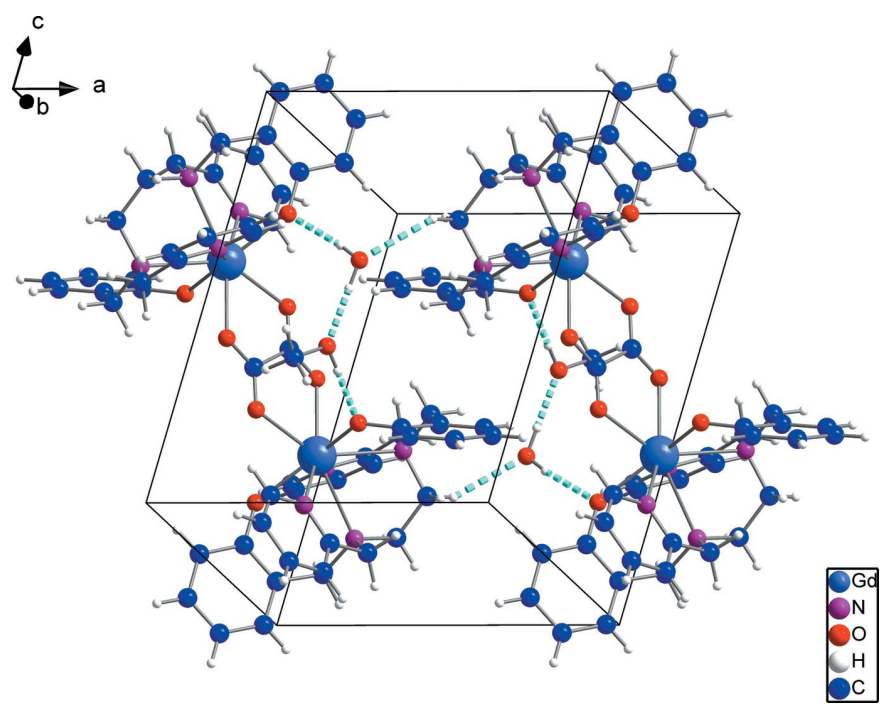

Figure 4

Representation of the dimeric molecules of $\mathbf{1}$ viewed approximately down the $b$ axis of the unit cell. The binuclear complexes are linked through medium-strength hydrogen bonds to solvating water and methanol molecules, and through weak $\mathrm{C} 1-\mathrm{H} 1 B \cdots \mathrm{O} 1 W^{\mathrm{ii}}-\mathrm{H} \cdots \mathrm{O}_{\text {phenolate }}$ interactions to one another [symmetry code: (ii) $-x+1,-y+1,-z+1$.]. 
concerned, with $\mathrm{O} 1 \mathrm{~W}$ acting as a potential electron-density acceptor, and are larger than $3.1 \AA$ for $\mathrm{O} \cdots \mathrm{O} 30$ (numbering scheme in Fig. 3). For 2, in turn, the corresponding distances are longer than for 1 at $2.88-3.84 \AA$ for $\mathrm{O} \cdots \mathrm{O} 1 \mathrm{~W}$, and even larger $(>4.6 \AA)$ for $\mathrm{O} \cdots \mathrm{O} 30$. On the other hand, any possible interaction involving the phenolate oxygen atoms would be very weak, with the shortest $\mathrm{O} \cdots \mathrm{O}$ contact with the disordered solvents being longer than $4.0 \AA$.

\section{Database survey}

Examples of mononuclear lanthanide(III) complexes with bbpen $^{2-}$ and related ligands appear in the literature (Molloy et al., 2017; Liu et al., 2016; Yamada et al., 2016; Gregório et al., 2015; Qin et al., 2014; Yamada et al., 2010; Morss \& Rogers, 1997). Binuclear structures with these hexadentate ligands have been reported by Chatterton et al. (2005), and by Setyawati et al. (2000).

\section{Synthesis and crystallization}

$L n \mathrm{Cl}_{3} \cdot 6 \mathrm{H}_{2} \mathrm{O}\left(L n^{\mathrm{III}}=\mathrm{Gd}\right.$ or Dy $)$ and $\mathrm{K}_{2} \mathrm{C}_{2} \mathrm{O}_{4} \cdot \mathrm{H}_{2} \mathrm{O}$ were purchased from Aldrich and used without purification. $N, N^{\prime}$ Bis(2-hydroxybenzyl)- $N, N^{\prime}$-bis(pyridin-2-ylmethyl)ethylenediamine $\left(\mathrm{H}_{2}\right.$ bbpen) (Neves et al., 1992) and the [ $\ln$ (bbpen)Cl] precursors, with $L n=\mathrm{Gd}$ or Dy (Liu et al., 2016), were prepared using adapted procedures described in the literature. Methanol and diethyl ether (Vetec) were used without treatment. Ultrapure water (Milli-Q, Millipore type 1, resistivity of $18.2 \mathrm{M} \Omega \mathrm{cm}$ at $298 \mathrm{~K}$ ) was employed as described below.

Synthesis of $\left[\{\mathrm{Gd}(\mathrm{bbpen})\}_{2}(\mu-\mathrm{ox})\right] \cdot 4 \mathrm{CH}_{3} \mathrm{OH} \cdot 4 \mathrm{H}_{2} \mathrm{O}$ (compound 1)

A solution of $8.11 \mathrm{mg}(0.0440 \mathrm{mmol})$ of $\mathrm{K}_{2} \mathrm{C}_{2} \mathrm{O}_{4} \cdot \mathrm{H}_{2} \mathrm{O}$ in $1.0 \mathrm{ml}$ of water was slowly added to a methanol solution of $61.1 \mathrm{mg}(0.0947 \mathrm{mmol})$ of $[\mathrm{Gd}($ bbpen $) \mathrm{Cl}]$. The colourless reaction mixture was stirred at room temperature for $c a 5 \mathrm{~min}$, and was then cooled down to $277 \mathrm{~K}$ to give block-shaped colourless crystals after four days. These were isolated by filtration, washed with diethyl ether and dried. Total yield: $49.0 \mathrm{mg}(68.6 \%)$ based on the $\left[\{\mathrm{Gd}(\text { bbpen })\}_{2}(\mu-\right.$ ox)] $4 \mathrm{CH}_{3} \mathrm{OH} \cdot 4 \mathrm{H}_{2} \mathrm{O}$ formulation, compound 1. FTIR (emulsion in mineral oil): $3362,3198[s, v(\mathrm{OH})] ; 1655\left[s, v(\mathrm{CO})_{\mathrm{ox}}\right]$; $1590,1568[s, v(\mathrm{C}=\mathrm{N})+v(\mathrm{C}=\mathrm{C})], 1290\left[s, v(\mathrm{CO})_{\text {phenolate }}\right]$, 762 and $768\left[\mathrm{~m}, \delta(\mathrm{C}-\mathrm{H})_{\mathrm{Ar}+\mathrm{py}}\right]$. Product $\mathbf{1}$ is soluble in acetonitrile, 1,2-dimethoxyethane (dme), dichloromethane and tetrahydrofuran. Elemental analysis: calculated for $\mathbf{1}$ $\left(\mathrm{C}_{62} \mathrm{H}_{80} \mathrm{Gd}_{2} \mathrm{~N}_{8} \mathrm{O}_{16}\right)$ C 49.39, H 5.35, N 7.43\%. Found: C 48.56, H 5.49, N 7.45\%.

\section{Synthesis of $\left[\{\text { Dy }(\text { bbpen })\}_{2}(\mu-o x)\right] \cdot 4 \mathrm{CH}_{3} \mathrm{OH} \cdot 4 \mathrm{H}_{2} \mathrm{O}$ (compound 2)}

A mixture of $61.0 \mathrm{mg}(0.0938 \mathrm{mmol})$ of [Dy(bbpen $) \mathrm{Cl}]$ in $9.0 \mathrm{ml}$ of methanol and $8.90 \mathrm{mg} \quad(0.0483 \mathrm{mmol})$ of $\mathrm{K}_{2} \mathrm{C}_{2} \mathrm{O}_{4} \cdot \mathrm{H}_{2} \mathrm{O}$ in $1.0 \mathrm{ml}$ of water was prepared as described for 1. The resulting solution was cooled at $277 \mathrm{~K}$ to produce colourless block-shaped crystals, which were recovered by filtration and washed with diethyl ether. Total yield: $53.9 \mathrm{mg}$ $(75.7 \%)$ based on the $\left.[\{\text { Dy (bbpen })\}_{2}(\mu-\mathrm{ox})\right] \cdot 4 \mathrm{CH}_{3} \mathrm{OH} \cdot 4 \mathrm{H}_{2} \mathrm{O}$ formulation, compound 2. FTIR (emulsion in mineral oil): 3363, $3198[s, v(\mathrm{OH})], 1590\left[s, v(\mathrm{CO})_{\mathrm{ox}}\right] ; 1570(\mathrm{~m}), 1481(\mathrm{~s})$, $1459[s, v(\mathrm{C}=\mathrm{N})+v(\mathrm{C}=\mathrm{C})] ; 1290\left[s, v(\mathrm{CO})_{\mathrm{Ph}}\right], 762$ and 768 $\left[m, \delta(\mathrm{C}-\mathrm{H})_{\mathrm{Ar}+\mathrm{py}}\right]$. The product solubility is similar to that described for $\mathbf{1}$. Elemental analysis: calculated for $\mathbf{2}$ $\left(\mathrm{C}_{62} \mathrm{H}_{80} \mathrm{Dy}_{2} \mathrm{~N}_{8} \mathrm{O}_{16}\right)$ C 49.04, H 5.31, N 7.38\%. Found: C 49.02, H 5.71, N 7.56\%.

\section{Refinement}

Crystal data, data collection and structure refinement details for the two structures are summarized in Table 3. Both $\mathbf{1}$ and $\mathbf{2}$ showed high susceptibility to the loss of the crystallization solvent molecules once removed from the mother liquor. Hydrogen atoms in $\mathbf{1}$ and $\mathbf{2}$ were included in idealized positions with methyl, methylene and aromatic $\mathrm{C}-\mathrm{H}$ distances set at $0.98,0.99$ and $0.95 \AA$, respectively, and $\mathrm{O}-\mathrm{H}$ at $0.84 \AA$ and refined as riding with $U_{\text {iso }}(\mathrm{H})=1.2-1.5 U_{\text {eq }}(\mathrm{C}, \mathrm{O})$. Hydrogen atoms on the water molecules were located in differenceFourier maps and were refined with distance restraints (DFIX $\mathrm{O}-\mathrm{H}=0.82 \AA$ for $\mathbf{1}$ and $\mathbf{2}$, DANG $=1.45 \AA$ for $\mathbf{2}$ ).

Both structures present four methanol and four water molecules per unit cell; two of each were treated as diffuse contribution to the overall scattering without specific atom positions and were eventually removed by the use of the SQUEEZE procedure in PLATON (Spek, 2015). The proposed identity of these highly disordered molecules as ' $2 \mathrm{H}_{2} \mathrm{O}+2 \mathrm{MeOH}$ ' per unit cell finds support in the total calculated count of 58 and 59 electrons provided by SQUEEZE for $\mathbf{1}$ and $\mathbf{2}$, respectively, as compared with the expected count of 56 electrons. The volume of the void filled by the disordered solvent amounts to 269 and $260 \AA^{3}$ for $\mathbf{1}$ and 2 , respectively, and corresponds to $16.0-16.5 \%$ of the unit cell, in very good agreement with the volume expected for small molecules such as water and methanol. The ratio between the total solvent-accessible void volume and the experimental electron count is of $c a 4.5 \AA^{3}$ per electron.

\section{Acknowledgements}

GAB and JFS thank the Conselho Nacional de Desenvolvimento Científico e Tecnológico (CNPq) and Coordenação de Aperfeiçoamento de Pessoal de Nível Superior (CAPES) for fellowships. The authors thank $\operatorname{Dr}$ David L. Hughes (University of East Anglia, UK) for training and discussions, and the late Professor Sueli M. Drechsel for helpful suggestions.

\section{Funding information}

Funding for this research was provided by: Fundação Araucária (grant No. 283/2014 - protocol 37509); Conselho Nacional de Desenvolvimento Científico e Tecnológico, CNPq (grant No. 308426/2016-9); Coordenação de Aperfeiçoamento de Pessoal de Nível Superior, CAPES (grant No. 001). 
Table 3

Experimental details.

1

Crystal data

Chemical formula

$M_{\mathrm{r}}$

Crystal system, space group

Temperature $(\mathrm{K})$

$a, b, c(\AA)$

$\alpha, \beta, \gamma\left(^{\circ}\right)$

$V\left(\AA^{3}\right)$

Z

Radiation type

$\mu\left(\mathrm{mm}^{-1}\right)$

Crystal size (mm)

Data collection

Diffractometer

Absorption correction

$T_{\min }, T_{\max }$

No. of measured, independent and

observed $[I>2 \sigma(I)]$ reflections

$R_{\text {int }}$

$(\sin \theta / \lambda)_{\max }\left(\AA^{-1}\right)$

Refinement

$R\left[F^{2}>2 \sigma\left(F^{2}\right)\right], w R\left(F^{2}\right), S$

No. of reflections

No. of parameters

No. of restraints

$\mathrm{H}$-atom treatment

$\Delta \rho_{\max }, \Delta \rho_{\min }\left(\mathrm{e} \AA^{-3}\right)$

\author{
$\left[\mathrm{Gd}_{2}\left(\mathrm{C}_{28} \mathrm{H}_{28} \mathrm{~N}_{4} \mathrm{O}_{2}\right)_{2}\left(\mathrm{C}_{2} \mathrm{O}_{4}\right)\right] \cdot 4 \mathrm{CH}_{4} \mathrm{O} \cdot 4 \mathrm{H}_{2} \mathrm{O}$ \\ 1507.84 \\ Triclinic, $P \overline{1}$ \\ 100 \\ 9.8778 (11), 12.8720 (16), 14.8025 (18) \\ 69.092 (4), 74.786 (4), 70.324 (4) \\ $1633.7(3)$ \\ 1 \\ Mo $K \alpha$ \\ 2.08 \\ $0.30 \times 0.28 \times 0.15$
}

Bruker D8 Venture/Photon 100 CMOS

Multi-scan (SADABS; Krause et al., 2015)

$0.613,0.746$

$101878,7108,6476$

0.052

0.639

$0.020,0.046,1.07$

7108

380

2

$\mathrm{H}$ atoms treated by a mixture of independent

and constrained refinement

$1.20,-0.55$
2

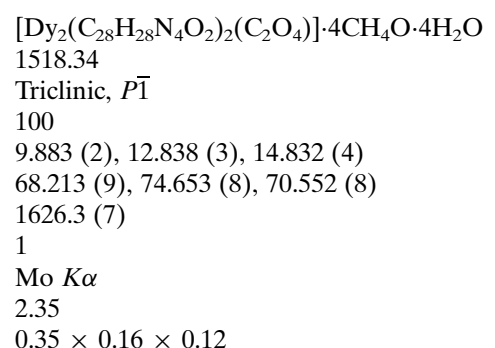

Bruker D8 Venture/Photon 100 CMOS Multi-scan (SADABS; Krause et al., 2015) $0.629,0.746$ $93133,7091,6385$

0.059

0.639

$0.025,0.060,1.06$

7091

380

3

$\mathrm{H}$ atoms treated by a mixture of independent and constrained refinement $1.94,-0.63$

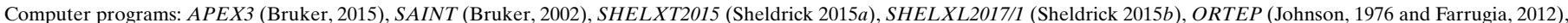
DIAMOND (Brandenburg, 2006), SHELXL97 (Sheldrick, 2008) and WinGX (Farrugia, 1999, 2012).

\section{References}

Brandenburg, K. (2006). DIAMOND. Crystal Impact GbR, Bonn, Germany.

Bruker (2002). SAINT. Bruker AXS Inc., Madison, Wisconsin, USA. Bruker (2015). APEX3. Bruker AXS Inc., Madison, Wisconsin, USA.

Chatterton, N. Y., Bretonnière, J., Pécaut, J. \& Mazzanti, M. (2005). Angew. Chem. Int. Ed. 44, 7595-7598.

Farrugia, L. J. (1999). J. Appl. Cryst. 32, 837-838.

Farrugia, L. J. (2012). J. Appl. Cryst. 45, 849-854.

Feng, X., Chen, J., Wang, L., Xie, S.-Y., Yang, S., Huo, S. \& Ng, S. (2014). CrystEngComm, 16, 1334-1343.

Gregório, T., Rüdiger, A. L., Nunes, G. G., Soares, J. F. \& Hughes, D. L. (2015). Acta Cryst. E71, 65-68.

Ishikawa, N., Sugita, M., Ishikawa, T., Koshihara, S. \& Kaizu, Y. (2003). J. Am. Chem. Soc. 125, 8694-8695.

Johnson, C. K. (1976). ORTEPII. Report ORNL-5138. Oak Ridge National Laboratory, Tennessee, USA.

Krause, L., Herbst-Irmer, R., Sheldrick, G. M. \& Stalke, D. (2015). J. Appl. Cryst. 48, 3-10.

Krishnamurty, K. V. \& Harris, G. M. (1960). J. Phys. Chem. 64, 346349.

Liu, J., Chen, Y.-C., Liu, J.-L., Vieru, V., Ungur, L., Jia, J.-H., Chibotaru, L. F., Lan, Y., Wernsdorfer, W., Gao, S., Chen, X.-M. \& Tong, M.-L. (2016). J. Am. Chem. Soc. 138, 5441-5450.

Llunell, M., Casanova, D., Cirera, J., Bofill, J. M., Alemany, P., Alvarez, S., Pinsky, M. \& Avnir, D. (2005). SHAPE. University of Barcelona and The Hebrew University of Jerusalem, Barcelona, Spain.
Molloy, J., Jarjayes, O., Philouze, C., Fedele, L., Imbert, D. \& Thomas, F. (2017). Chem. Commun. 53, 605-608.

Morss, L. \& Rogers, R. (1997). Inorg. Chim. Acta, 255, 193197.

Neves, A., Erthal, S. M. D., Vencato, I., Ceccato, A. S., Mascarenhas, Y. P., Nascimento, O. R., Horner, M. \& Batista, A. A. (1992). Inorg. Chem. 31, 4749-4755.

Piquer, L. P. \& Sañudo, E. C. (2015). Dalton Trans. 44, 8771-8780.

Qin, J., Wang, P., Li, Q., Zhang, Y., Yuan, D. \& Yao, Y. (2014). Chem. Commun. 50, 10952-10955.

Setyawati, I. A., Liu, S., Rettig, S. J. \& Orvig, C. (2000). Inorg. Chem. 39, 496-507.

Shannon, R. D. (1976). Acta Cryst. A32, 751-767.

Sheldrick, G. M. (2008). Acta Cryst. A64, 112-122.

Sheldrick, G. M. (2015a). Acta Cryst. A71, 3-8.

Sheldrick, G. M. (2015b). Acta Cryst. C71, 3-8.

Spek, A. L. (2015). Acta Cryst. C71, 9-18.

Xu, G. F., Gamez, P., Tang, J., Clérac, R., Guo, Y. N. \& Guo, Y. (2012). Inorg. Chem. 51, 5693-5698.

Xu, G.-F., Wang, Q.-L., Gamez, P., Ma, Y., Clérac, R., Tang, J., Yan, S.-P., Cheng, P. \& Liao, D.-Z. (2010). Chem. Commun. 46, 15061508.

Yamada, Y., Koori, D., Mori, K. \& Oshikawa, Y. (2016). J. Coord. Chem. 69, 3735-3744.

Yamada, Y., Takenouchi, S. I., Miyoshi, Y. \& Okamoto, K. I. (2010). J. Coord. Chem. 63, 996-1012.

Zhang, S., Ke, H., Liu, X., Wei, Q., Xie, G. \& Chen, S. (2015). Chem. Commun. 51, 15188-15191. 


\section{supporting information}

Acta Cryst. (2019). E75, 418-422 [https://doi.org/10.1107/S2056989019002998]

Crystal structures of binuclear complexes of gadolinium(III) and dysprosium(III) with oxalate bridges and chelating $N, N^{\prime}$-bis(2-oxidobenzyl)- $N, N^{\prime}$-bis(pyridin-2ylmethyl)ethylenediamine (bbpen ${ }^{2-}$ )

\section{Guilherme Augusto Barbosa, Francielli Sousa Santana, Giovana Gioppo Nunes and Jaísa Fernandes Soares}

Computing details

For both structures, data collection: APEX3 (Bruker, 2015); cell refinement: SAINT (Bruker, 2002); data reduction: SAINT (Bruker, 2002); program(s) used to solve structure: SHELXT2015 (Sheldrick 2015a); program(s) used to refine structure: SHELXL2017/1 (Sheldrick 2015b); molecular graphics: ORTEP (Johnson, 1976 and Farrugia, 2012) and DIAMOND (Brandenburg, 2006). Software used to prepare material for publication: SHELXL97 (Sheldrick, 2008) and WinGX (Farrugia, 1999, 2012) for (1); SHELXL97 (Sheldrick, 2008) and WinGX (Farrugia, 1999, Farrugia, 2012) for (2).

( $\mu$-Oxalato)bis $\left\{\left[N_{,}, N^{\prime}\right.\right.$-bis(2-oxidobenzyl- $\left.\kappa O\right)-N_{,} N^{\prime}$-bis(pyridin-2-ylmethyl- $\kappa N$ ) ethylenediamine$\left.\kappa^{2} N, N^{\prime}\right]$ gadolinium(III)\}-methanol-water (1/4/4) (1)

Crystal data

$\left[\mathrm{Gd}_{2}\left(\mathrm{C}_{28} \mathrm{H}_{28} \mathrm{~N}_{4} \mathrm{O}_{2}\right)_{2}\left(\mathrm{C}_{2} \mathrm{O}_{4}\right)\right] \cdot 4 \mathrm{CH}_{4} \mathrm{O} \cdot 4 \mathrm{H}_{2} \mathrm{O}$

$M_{r}=1507.84$

Triclinic, $P \overline{1}$

$a=9.8778(11) \AA$

$b=12.8720(16) \AA$

$c=14.8025$ (18) $\AA$

$\alpha=69.092(4)^{\circ}$

$\beta=74.786(4)^{\circ}$

$\gamma=70.324(4)^{\circ}$

$V=1633.7(3) \AA^{3}$

Data collection

Bruker D8 Venture/Photon 100 CMOS diffractometer

Radiation source: fine-focus sealed tube Graphite monochromator

Detector resolution: 10.4167 pixels $\mathrm{mm}^{-1}$

$\varphi$ and $\omega$ scans

Absorption correction: multi-scan

(SADABS; Krause et al., 2015)

$T_{\min }=0.613, T_{\max }=0.746$
$Z=1$

$F(000)=764$

$D_{\mathrm{x}}=1.533 \mathrm{Mg} \mathrm{m}^{-3}$

Mo $K \alpha$ radiation, $\lambda=0.71073 \AA$

Cell parameters from 9519 reflections

$\theta=3.0-27.9^{\circ}$

$\mu=2.08 \mathrm{~mm}^{-1}$

$T=100 \mathrm{~K}$

Prism, colourless

$0.30 \times 0.28 \times 0.14 \mathrm{~mm}$

101878 measured reflections

7108 independent reflections

6476 reflections with $I>2 \sigma(I)$

$R_{\text {int }}=0.052$

$\theta_{\max }=27.0^{\circ}, \theta_{\min }=3.0^{\circ}$

$h=-12 \rightarrow 12$

$k=-16 \rightarrow 16$

$l=-18 \rightarrow 18$ 


\section{Refinement}

Refinement on $F^{2}$

Least-squares matrix: full

$R\left[F^{2}>2 \sigma\left(F^{2}\right)\right]=0.020$

$w R\left(F^{2}\right)=0.046$

$S=1.07$

7108 reflections

380 parameters

2 restraints

Primary atom site location: dual
Secondary atom site location: difference Fourier map

Hydrogen site location: mixed

$\mathrm{H}$ atoms treated by a mixture of independent and constrained refinement

$w=1 /\left[\sigma^{2}\left(F_{\mathrm{o}}^{2}\right)+(0.0132 P)^{2}+1.5506 P\right]$ where $P=\left(F_{\mathrm{o}}{ }^{2}+2 F_{\mathrm{c}}{ }^{2}\right) / 3$

$(\Delta / \sigma)_{\max }=0.001$

$\Delta \rho_{\max }=1.20 \mathrm{e} \AA^{-3}$

$\Delta \rho_{\min }=-0.55$ e $\AA^{-3}$

Special details

Geometry. All esds (except the esd in the dihedral angle between two 1.s. planes) are estimated using the full covariance matrix. The cell esds are taken into account individually in the estimation of esds in distances, angles and torsion angles; correlations between esds in cell parameters are only used when they are defined by crystal symmetry. An approximate (isotropic) treatment of cell esds is used for estimating esds involving 1.s. planes.

Fractional atomic coordinates and isotropic or equivalent isotropic displacement parameters $\left(\AA^{2}\right)$

\begin{tabular}{|c|c|c|c|c|}
\hline & $x$ & $y$ & $z$ & $U_{\text {iso }} * / U_{\text {eq }}$ \\
\hline Gd1 & $0.14723(2)$ & $0.64026(2)$ & $0.30503(2)$ & $0.02451(4)$ \\
\hline N1 & $0.3709(2)$ & $0.68776(16)$ & $0.33127(13)$ & $0.0308(4)$ \\
\hline N2 & $0.26964(19)$ & $0.76071(16)$ & $0.13842(13)$ & $0.0302(4)$ \\
\hline N3 & $0.0847(2)$ & $0.83950(16)$ & $0.33400(14)$ & $0.0345(4)$ \\
\hline N4 & $0.1895(2)$ & $0.56231(17)$ & $0.16178(14)$ & $0.0341(4)$ \\
\hline O1 & $0.32272(17)$ & 0.47047 (14) & $0.33368(13)$ & $0.0384(4)$ \\
\hline $\mathrm{O} 2$ & $-0.04416(16)$ & $0.74756(14)$ & $0.22275(11)$ & $0.0348(4)$ \\
\hline $\mathrm{O} 3$ & $0.10169(17)$ & $0.59622(14)$ & $0.47919(11)$ & $0.0326(3)$ \\
\hline O4 & $0.00913(17)$ & 0.48457 (14) & $0.62012(11)$ & $0.0317(3)$ \\
\hline $\mathrm{O} 30$ & $0.2560(3)$ & $0.2853(2)$ & $0.46553(17)$ & $0.0708(6)$ \\
\hline H30 & 0.279892 & 0.342451 & 0.422530 & $0.106^{*}$ \\
\hline $\mathrm{C} 1$ & $0.4553(3)$ & $0.7393(2)$ & $0.23546(17)$ & $0.0366(5)$ \\
\hline $\mathrm{H} 1 \mathrm{~A}$ & 0.526548 & 0.676065 & 0.211006 & $0.044 *$ \\
\hline H1B & 0.510810 & 0.783956 & 0.246233 & $0.044^{*}$ \\
\hline $\mathrm{C} 2$ & $0.3634(3)$ & $0.8170(2)$ & $0.15829(17)$ & $0.0356(5)$ \\
\hline $\mathrm{H} 2 \mathrm{~A}$ & 0.300546 & 0.885400 & 0.179071 & $0.043^{*}$ \\
\hline $\mathrm{H} 2 \mathrm{~B}$ & 0.428067 & 0.844484 & 0.096725 & $0.043 *$ \\
\hline C3 & $0.4722(3)$ & $0.5828(2)$ & $0.38753(18)$ & $0.0371(5)$ \\
\hline $\mathrm{H} 3 \mathrm{~A}$ & 0.545817 & 0.607522 & 0.403134 & $0.045^{*}$ \\
\hline $\mathrm{H} 3 \mathrm{~B}$ & 0.415669 & 0.547543 & 0.450400 & $0.045^{*}$ \\
\hline $\mathrm{C} 4$ & $0.5499(3)$ & 0.4925 (2) & $0.33652(18)$ & $0.0376(5)$ \\
\hline $\mathrm{C} 5$ & $0.7021(3)$ & $0.4568(3)$ & $0.3171(2)$ & $0.0530(7)$ \\
\hline H5 & 0.757587 & 0.492628 & 0.334115 & $0.064 *$ \\
\hline C6 & $0.7724(3)$ & $0.3699(3)$ & $0.2734(3)$ & $0.0662(9)$ \\
\hline H6 & 0.875802 & 0.345578 & 0.260954 & $0.079 *$ \\
\hline $\mathrm{C} 7$ & $0.6921(3)$ & 0.3189 (3) & $0.2482(2)$ & $0.0595(8)$ \\
\hline H7 & 0.740815 & 0.259677 & 0.217648 & $0.071 *$ \\
\hline C8 & $0.5402(3)$ & $0.3526(2)$ & $0.2666(2)$ & $0.0473(6)$ \\
\hline
\end{tabular}




\begin{tabular}{|c|c|c|c|c|}
\hline H8 & 0.485806 & 0.316915 & 0.248361 & $0.057^{*}$ \\
\hline C9 & $0.4686(3)$ & $0.4392(2)$ & $0.31219(17)$ & $0.0359(5)$ \\
\hline $\mathrm{C} 10$ & $0.1601(3)$ & $0.8573(2)$ & $0.08153(18)$ & $0.0361(5)$ \\
\hline $\mathrm{H} 10 \mathrm{~A}$ & 0.213640 & 0.898996 & 0.020397 & $0.043^{*}$ \\
\hline H10B & 0.109274 & 0.912135 & 0.120374 & $0.043^{*}$ \\
\hline $\mathrm{C} 11$ & $0.0468(2)$ & 0.82508 (19) & $0.05439(17)$ & $0.0327(5)$ \\
\hline $\mathrm{C} 12$ & $0.0333(3)$ & $0.8522(2)$ & $-0.04292(19)$ & $0.0419(6)$ \\
\hline H12 & 0.103411 & 0.884025 & -0.093012 & $0.050^{*}$ \\
\hline $\mathrm{C} 13$ & $-0.0798(3)$ & $0.8339(2)$ & $-0.0688(2)$ & $0.0483(7)$ \\
\hline H13 & -0.087322 & 0.852986 & -0.135819 & $0.058^{*}$ \\
\hline $\mathrm{C} 14$ & $-0.1812(3)$ & $0.7878(3)$ & $0.0040(2)$ & $0.0514(7)$ \\
\hline H14 & -0.260080 & 0.776115 & -0.012986 & $0.062 *$ \\
\hline $\mathrm{C} 15$ & $-0.1695(3)$ & $0.7584(2)$ & $0.1014(2)$ & $0.0438(6)$ \\
\hline H15 & -0.239531 & 0.725264 & 0.150492 & $0.053^{*}$ \\
\hline $\mathrm{C} 16$ & $-0.0560(2)$ & $0.77657(19)$ & $0.12913(17)$ & $0.0318(5)$ \\
\hline $\mathrm{C} 17$ & $0.3155(3)$ & $0.7667(2)$ & 0.39309 (19) & $0.0399(6)$ \\
\hline H17A & 0.393828 & 0.801043 & 0.388604 & $0.048^{*}$ \\
\hline H17B & 0.293976 & 0.720619 & 0.462140 & $0.048^{*}$ \\
\hline $\mathrm{C} 18$ & 0.1824 (3) & $0.8622(2)$ & $0.36726(18)$ & $0.0379(5)$ \\
\hline $\mathrm{C} 19$ & $0.1574(4)$ & $0.9676(3)$ & $0.3832(2)$ & $0.0555(7)$ \\
\hline H19 & 0.230010 & 0.983182 & 0.403804 & $0.067 *$ \\
\hline $\mathrm{C} 20$ & $0.0264(4)$ & $1.0490(3)$ & $0.3690(3)$ & $0.0643(9)$ \\
\hline $\mathrm{H} 20$ & 0.006686 & 1.120691 & 0.381254 & $0.077^{*}$ \\
\hline $\mathrm{C} 21$ & $-0.0753(3)$ & $1.0259(2)$ & $0.3370(2)$ & $0.0537(7)$ \\
\hline H21 & -0.167055 & 1.080485 & 0.327493 & $0.064 *$ \\
\hline $\mathrm{C} 22$ & $-0.0409(3)$ & $0.9211(2)$ & $0.31884(19)$ & $0.0427(6)$ \\
\hline $\mathrm{H} 22$ & -0.110029 & 0.906124 & 0.294343 & $0.051^{*}$ \\
\hline $\mathrm{C} 23$ & $0.3579(3)$ & $0.6806(2)$ & $0.08111(18)$ & $0.0379(5)$ \\
\hline $\mathrm{H} 23 \mathrm{~A}$ & 0.448335 & 0.634954 & 0.109017 & $0.046^{*}$ \\
\hline $\mathrm{H} 23 \mathrm{~B}$ & 0.386281 & 0.726014 & 0.012906 & $0.046^{*}$ \\
\hline $\mathrm{C} 24$ & 0.2797 (2) & $0.5989(2)$ & $0.07971(17)$ & $0.0339(5)$ \\
\hline $\mathrm{C} 25$ & $0.3037(3)$ & $0.5614(2)$ & $-0.00088(19)$ & $0.0468(6)$ \\
\hline H25 & 0.369457 & 0.587595 & -0.057776 & $0.056^{*}$ \\
\hline $\mathrm{C} 26$ & $0.2311(3)$ & $0.4857(3)$ & $0.0022(2)$ & $0.0563(8)$ \\
\hline $\mathrm{H} 26$ & 0.245896 & 0.459136 & -0.052756 & $0.068^{*}$ \\
\hline $\mathrm{C} 27$ & 0.1367 (3) & $0.4485(3)$ & $0.0856(2)$ & $0.0547(7)$ \\
\hline $\mathrm{H} 27$ & 0.084898 & 0.396546 & 0.089189 & $0.066^{*}$ \\
\hline $\mathrm{C} 28$ & $0.1195(3)$ & $0.4888(2)$ & $0.1637(2)$ & $0.0452(6)$ \\
\hline $\mathrm{H} 28$ & 0.054965 & 0.463138 & 0.221597 & $0.054 *$ \\
\hline $\mathrm{C} 29$ & $0.0323(2)$ & $0.52339(19)$ & $0.52893(15)$ & $0.0270(4)$ \\
\hline $\mathrm{C} 30$ & $0.1948(4)$ & $0.2316(3)$ & $0.4227(3)$ & $0.0712(10)$ \\
\hline $\mathrm{H} 30 \mathrm{~A}$ & 0.185641 & 0.156599 & 0.468653 & $0.107^{*}$ \\
\hline $\mathrm{H} 30 \mathrm{~B}$ & 0.258399 & 0.220344 & 0.361943 & $0.107 *$ \\
\hline $\mathrm{H} 30 \mathrm{C}$ & 0.098312 & 0.281001 & 0.408213 & $0.107^{*}$ \\
\hline $\mathrm{O} 1 \mathrm{~W}$ & $0.3109(2)$ & $0.2151(2)$ & $0.65369(19)$ & $0.0644(6)$ \\
\hline H1W & $0.236(3)$ & $0.229(3)$ & $0.694(2)$ & $0.058(10)^{*}$ \\
\hline $\mathrm{H} 2 \mathrm{~W}$ & $0.290(4)$ & $0.245(3)$ & $0.5939(16)$ & $0.080(13)^{*}$ \\
\hline
\end{tabular}


Atomic displacement parameters $\left(\AA^{2}\right)$

\begin{tabular}{|c|c|c|c|c|c|c|}
\hline & $U^{11}$ & $U^{22}$ & $U^{33}$ & $U^{12}$ & $U^{13}$ & $U^{23}$ \\
\hline Gd1 & $0.02416(5)$ & $0.02753(6)$ & $0.02339(6)$ & -0.01192 & -0.00064 (4) & -0.00698 \\
\hline N1 & $0.0324(10)$ & $0.0350(10)$ & $0.0268(9)$ & $-0.0153(8)$ & $-0.0065(7)$ & $-0.0041(8)$ \\
\hline N2 & $0.0287(9)$ & $0.0350(10)$ & $0.0276(10)$ & $-0.0149(8)$ & $-0.0022(7)$ & $-0.0059(8)$ \\
\hline N3 & $0.0390(11)$ & $0.0297(10)$ & $0.0347(11)$ & $-0.0105(8)$ & $-0.0042(8)$ & $-0.0096(8)$ \\
\hline N4 & $0.0412(11)$ & $0.0324(10)$ & $0.0293(10)$ & $-0.0113(8)$ & $-0.0030(8)$ & $-0.0104(8)$ \\
\hline $\mathrm{O} 1$ & $0.0309(8)$ & $0.0337(9)$ & $0.0529(11)$ & $-0.0067(7)$ & -0.0099 (7) & $-0.0151(8)$ \\
\hline $\mathrm{O} 2$ & $0.0288(8)$ & $0.0438(9)$ & $0.0317(9)$ & $-0.0098(7)$ & $-0.0044(6)$ & $-0.0112(7)$ \\
\hline $\mathrm{O} 3$ & $0.0387(9)$ & $0.0427(9)$ & $0.0272(8)$ & $-0.0279(7)$ & $0.0007(6)$ & $-0.0112(7)$ \\
\hline O4 & $0.0386(8)$ & $0.0444(9)$ & $0.0215(8)$ & $-0.0259(7)$ & $-0.0012(6)$ & $-0.0097(7)$ \\
\hline $\mathrm{O} 30$ & 0.1006 (19) & $0.0616(15)$ & $0.0601(14)$ & $-0.0466(14)$ & $-0.0135(13)$ & $-0.0058(11)$ \\
\hline $\mathrm{C} 1$ & $0.0330(12)$ & 0.0437 (14) & $0.0358(13)$ & $-0.0222(10)$ & $-0.0053(10)$ & $-0.0042(11)$ \\
\hline $\mathrm{C} 2$ & $0.0369(12)$ & $0.0364(13)$ & $0.0316(12)$ & $-0.0202(10)$ & $-0.0003(9)$ & $-0.0022(10)$ \\
\hline $\mathrm{C} 3$ & $0.0353(12)$ & $0.0414(14)$ & $0.0365(13)$ & $-0.0137(10)$ & $-0.0160(10)$ & $-0.0032(11)$ \\
\hline $\mathrm{C} 4$ & $0.0310(12)$ & $0.0391(13)$ & $0.0351(13)$ & $-0.0071(10)$ & $-0.0087(10)$ & $-0.0019(10)$ \\
\hline $\mathrm{C} 5$ & $0.0336(14)$ & $0.0506(17)$ & 0.0624 (19) & $-0.0070(12)$ & $-0.0130(12)$ & $-0.0027(14)$ \\
\hline C6 & $0.0333(15)$ & $0.062(2)$ & $0.073(2)$ & $0.0010(14)$ & $0.0003(14)$ & $-0.0043(17)$ \\
\hline $\mathrm{C} 7$ & $0.0556(18)$ & $0.0416(16)$ & $0.0500(17)$ & $0.0096(14)$ & $0.0026(14)$ & $-0.0065(13)$ \\
\hline $\mathrm{C} 8$ & 0.0543 (16) & $0.0352(14)$ & $0.0424(15)$ & $-0.0020(12)$ & $-0.0084(12)$ & $-0.0091(12)$ \\
\hline $\mathrm{C} 9$ & $0.0345(12)$ & $0.0324(12)$ & $0.0318(12)$ & $-0.0021(10)$ & $-0.0082(9)$ & $-0.0033(10)$ \\
\hline $\mathrm{C} 10$ & $0.0411(13)$ & $0.0301(12)$ & $0.0344(13)$ & $-0.0143(10)$ & $-0.0081(10)$ & $-0.0006(10)$ \\
\hline $\mathrm{C} 11$ & $0.0339(12)$ & $0.0268(11)$ & $0.0347(12)$ & $-0.0047(9)$ & $-0.0081(9)$ & $-0.0073(9)$ \\
\hline $\mathrm{C} 12$ & $0.0500(15)$ & $0.0333(13)$ & $0.0361(13)$ & $-0.0061(11)$ & $-0.0109(11)$ & $-0.0047(11)$ \\
\hline $\mathrm{C} 13$ & 0.0592 (17) & $0.0458(15)$ & $0.0416(15)$ & $-0.0034(13)$ & $-0.0221(13)$ & $-0.0149(12)$ \\
\hline $\mathrm{C} 14$ & $0.0476(16)$ & $0.0574(18)$ & 0.0617 (19) & $-0.0079(13)$ & $-0.0215(14)$ & $-0.0290(15)$ \\
\hline $\mathrm{C} 15$ & $0.0333(12)$ & $0.0560(16)$ & $0.0490(15)$ & $-0.0134(11)$ & $-0.0070(11)$ & $-0.0220(13)$ \\
\hline $\mathrm{C} 16$ & 0.0293 (11) & $0.0301(11)$ & $0.0355(12)$ & $-0.0030(9)$ & $-0.0067(9)$ & $-0.0127(10)$ \\
\hline $\mathrm{C} 17$ & $0.0421(13)$ & $0.0437(14)$ & $0.0435(14)$ & $-0.0163(11)$ & $-0.0125(11)$ & $-0.0156(12)$ \\
\hline $\mathrm{C} 18$ & $0.0475(14)$ & $0.0371(13)$ & 0.0337 (13) & $-0.0179(11)$ & $-0.0023(10)$ & $-0.0127(10)$ \\
\hline C19 & $0.074(2)$ & $0.0444(16)$ & 0.0619 (19) & $-0.0200(15)$ & $-0.0167(16)$ & $-0.0240(14)$ \\
\hline $\mathrm{C} 20$ & $0.090(2)$ & 0.0409 (16) & $0.070(2)$ & $-0.0141(16)$ & $-0.0138(18)$ & $-0.0284(16)$ \\
\hline $\mathrm{C} 21$ & $0.0620(18)$ & $0.0353(14)$ & 0.0529 (17) & $0.0005(13)$ & $-0.0088(14)$ & $-0.0135(13)$ \\
\hline $\mathrm{C} 22$ & $0.0426(14)$ & $0.0375(14)$ & $0.0446(15)$ & $-0.0077(11)$ & $-0.0046(11)$ & $-0.0128(11)$ \\
\hline $\mathrm{C} 23$ & $0.0324(12)$ & 0.0463 (14) & $0.0311(12)$ & $-0.0132(10)$ & $0.0023(9)$ & $-0.0097(11)$ \\
\hline $\mathrm{C} 24$ & $0.0326(12)$ & $0.0341(12)$ & $0.0291(12)$ & $0.0005(9)$ & $-0.0064(9)$ & $-0.0102(10)$ \\
\hline $\mathrm{C} 25$ & $0.0471(15)$ & $0.0544(17)$ & $0.0348(14)$ & $-0.0071(13)$ & $0.0002(11)$ & $-0.0192(12)$ \\
\hline $\mathrm{C} 26$ & 0.0669 (19) & 0.0640 (19) & $0.0470(17)$ & $-0.0125(15)$ & $-0.0055(14)$ & $-0.0342(15)$ \\
\hline $\mathrm{C} 27$ & 0.0655 (19) & $0.0513(17)$ & $0.0620(19)$ & $-0.0211(14)$ & $-0.0082(15)$ & $-0.0301(15)$ \\
\hline $\mathrm{C} 28$ & $0.0586(16)$ & $0.0432(15)$ & $0.0410(14)$ & $-0.0220(13)$ & $-0.0014(12)$ & $-0.0178(12)$ \\
\hline $\mathrm{C} 29$ & $0.0263(10)$ & $0.0321(11)$ & $0.0270(11)$ & $-0.0126(9)$ & $-0.0008(8)$ & $-0.0119(9)$ \\
\hline $\mathrm{C} 30$ & $0.094(3)$ & 0.0534 (19) & $0.076(2)$ & $-0.0322(18)$ & $0.001(2)$ & $-0.0293(18)$ \\
\hline $\mathrm{O} 1 \mathrm{~W}$ & $0.0331(11)$ & 0.1015 (19) & $0.0682(16)$ & $-0.0197(11)$ & $-0.0019(11)$ & $-0.0393(15)$ \\
\hline
\end{tabular}


Geometric parameters $\left(\AA,{ }^{\circ}\right)$

\begin{tabular}{|c|c|c|c|}
\hline $\mathrm{Gd} 1-\mathrm{O} 1$ & $2.2659(16)$ & $\mathrm{C} 10-\mathrm{C} 11$ & $1.501(3)$ \\
\hline $\mathrm{Gd} 1-\mathrm{O} 2$ & $2.2835(16)$ & $\mathrm{C} 10-\mathrm{H} 10 \mathrm{~A}$ & 0.9900 \\
\hline $\mathrm{Gd} 1-\mathrm{O} 3$ & $2.3855(15)$ & $\mathrm{C} 10-\mathrm{H} 10 \mathrm{~B}$ & 0.9900 \\
\hline $\mathrm{Gd} 1-\mathrm{O} 4^{\mathrm{i}}$ & $2.3869(14)$ & $\mathrm{C} 11-\mathrm{C} 12$ & $1.388(3)$ \\
\hline Gd1—N4 & 2.5394 (19) & $\mathrm{C} 11-\mathrm{C} 16$ & $1.410(3)$ \\
\hline $\mathrm{Gd} 1-\mathrm{N} 3$ & $2.5926(19)$ & $\mathrm{C} 12-\mathrm{C} 13$ & $1.384(4)$ \\
\hline $\mathrm{Gd} 1-\mathrm{N} 2$ & $2.6299(18)$ & $\mathrm{C} 12-\mathrm{H} 12$ & 0.9500 \\
\hline $\mathrm{Gd} 1-\mathrm{N} 1$ & $2.6334(18)$ & $\mathrm{C} 13-\mathrm{C} 14$ & $1.376(4)$ \\
\hline $\mathrm{N} 1-\mathrm{C} 17$ & $1.482(3)$ & $\mathrm{C} 13-\mathrm{H} 13$ & 0.9500 \\
\hline $\mathrm{N} 1-\mathrm{C} 1$ & $1.492(3)$ & $\mathrm{C} 14-\mathrm{C} 15$ & $1.379(4)$ \\
\hline $\mathrm{N} 1-\mathrm{C} 3$ & $1.498(3)$ & C14-H14 & 0.9500 \\
\hline $\mathrm{N} 2-\mathrm{C} 23$ & $1.475(3)$ & $\mathrm{C} 15-\mathrm{C} 16$ & $1.403(3)$ \\
\hline $\mathrm{N} 2-\mathrm{C} 2$ & $1.489(3)$ & C15-H15 & 0.9500 \\
\hline $\mathrm{N} 2-\mathrm{C} 10$ & $1.500(3)$ & $\mathrm{C} 17-\mathrm{C} 18$ & $1.492(4)$ \\
\hline $\mathrm{N} 3-\mathrm{C} 22$ & $1.339(3)$ & C17-H17A & 0.9900 \\
\hline N3-C18 & $1.343(3)$ & C17-H17B & 0.9900 \\
\hline $\mathrm{N} 4-\mathrm{C} 28$ & $1.336(3)$ & C18-C19 & $1.389(4)$ \\
\hline $\mathrm{N} 4-\mathrm{C} 24$ & $1.340(3)$ & $\mathrm{C} 19-\mathrm{C} 20$ & $1.374(5)$ \\
\hline $\mathrm{O} 1-\mathrm{C} 9$ & $1.342(3)$ & C19-H19 & 0.9500 \\
\hline $\mathrm{O} 2-\mathrm{C} 16$ & $1.327(3)$ & $\mathrm{C} 20-\mathrm{C} 21$ & $1.369(5)$ \\
\hline $\mathrm{O} 3-\mathrm{C} 29$ & $1.252(3)$ & $\mathrm{C} 20-\mathrm{H} 20$ & 0.9500 \\
\hline $\mathrm{O} 4-\mathrm{C} 29$ & $1.248(3)$ & $\mathrm{C} 21-\mathrm{C} 22$ & $1.382(4)$ \\
\hline $\mathrm{O} 30-\mathrm{C} 30$ & $1.429(4)$ & $\mathrm{C} 21-\mathrm{H} 21$ & 0.9500 \\
\hline $\mathrm{O} 30-\mathrm{H} 30$ & 0.8400 & $\mathrm{C} 22-\mathrm{H} 22$ & 0.9500 \\
\hline $\mathrm{C} 1-\mathrm{C} 2$ & $1.499(3)$ & $\mathrm{C} 23-\mathrm{C} 24$ & $1.508(3)$ \\
\hline $\mathrm{C} 1-\mathrm{H} 1 \mathrm{~A}$ & 0.9900 & $\mathrm{C} 23-\mathrm{H} 23 \mathrm{~A}$ & 0.9900 \\
\hline $\mathrm{C} 1-\mathrm{H} 1 \mathrm{~B}$ & 0.9900 & $\mathrm{C} 23-\mathrm{H} 23 \mathrm{~B}$ & 0.9900 \\
\hline $\mathrm{C} 2-\mathrm{H} 2 \mathrm{~A}$ & 0.9900 & $\mathrm{C} 24-\mathrm{C} 25$ & $1.377(3)$ \\
\hline $\mathrm{C} 2-\mathrm{H} 2 \mathrm{~B}$ & 0.9900 & $\mathrm{C} 25-\mathrm{C} 26$ & $1.375(4)$ \\
\hline $\mathrm{C} 3-\mathrm{C} 4$ & $1.494(4)$ & $\mathrm{C} 25-\mathrm{H} 25$ & 0.9500 \\
\hline $\mathrm{C} 3-\mathrm{H} 3 \mathrm{~A}$ & 0.9900 & $\mathrm{C} 26-\mathrm{C} 27$ & $1.377(4)$ \\
\hline C $3-\mathrm{H} 3 \mathrm{~B}$ & 0.9900 & $\mathrm{C} 26-\mathrm{H} 26$ & 0.9500 \\
\hline $\mathrm{C} 4-\mathrm{C} 9$ & $1.393(4)$ & $\mathrm{C} 27-\mathrm{C} 28$ & $1.379(4)$ \\
\hline $\mathrm{C} 4-\mathrm{C} 5$ & $1.399(3)$ & $\mathrm{C} 27-\mathrm{H} 27$ & 0.9500 \\
\hline $\mathrm{C} 5-\mathrm{C} 6$ & $1.382(5)$ & $\mathrm{C} 28-\mathrm{H} 28$ & 0.9500 \\
\hline $\mathrm{C} 5-\mathrm{H} 5$ & 0.9500 & $\mathrm{C} 29-\mathrm{C} 29^{\mathrm{i}}$ & $1.554(4)$ \\
\hline $\mathrm{C} 6-\mathrm{C} 7$ & $1.371(5)$ & $\mathrm{C} 30-\mathrm{H} 30 \mathrm{~A}$ & 0.9800 \\
\hline C6-H6 & 0.9500 & $\mathrm{C} 30-\mathrm{H} 30 \mathrm{~B}$ & 0.9800 \\
\hline $\mathrm{C} 7-\mathrm{C} 8$ & $1.395(4)$ & $\mathrm{C} 30-\mathrm{H} 30 \mathrm{C}$ & 0.9800 \\
\hline $\mathrm{C} 7-\mathrm{H} 7$ & 0.9500 & $\mathrm{O} 1 \mathrm{~W}-\mathrm{H} 1 \mathrm{~W}$ & $0.831(18)$ \\
\hline $\mathrm{C} 8-\mathrm{C} 9$ & $1.398(4)$ & $\mathrm{O} 1 \mathrm{~W}-\mathrm{H} 2 \mathrm{~W}$ & $0.879(18)$ \\
\hline $\mathrm{C} 8-\mathrm{H} 8$ & 0.9500 & & \\
\hline $\mathrm{O} 1-\mathrm{Gd} 1-\mathrm{O} 2$ & $144.65(6)$ & $\mathrm{C} 7-\mathrm{C} 8-\mathrm{H} 8$ & 120.3 \\
\hline $\mathrm{O} 1-\mathrm{Gd} 1-\mathrm{O} 3$ & $83.85(6)$ & $\mathrm{C} 9-\mathrm{C} 8-\mathrm{H} 8$ & 120.3 \\
\hline $\mathrm{O} 2-\mathrm{Gd} 1-\mathrm{O} 3$ & $117.78(6)$ & $\mathrm{O} 1-\mathrm{C} 9-\mathrm{C} 4$ & $119.8(2)$ \\
\hline
\end{tabular}




\begin{tabular}{|c|c|c|c|}
\hline $\mathrm{O} 1-\mathrm{Gd} 1-\mathrm{O} 4^{\mathrm{i}}$ & $82.26(6)$ & $\mathrm{O} 1-\mathrm{C} 9-\mathrm{C} 8$ & $120.6(2)$ \\
\hline $\mathrm{O} 2-\mathrm{Gd} 1-\mathrm{O} 4^{\mathrm{i}}$ & $81.09(6)$ & $\mathrm{C} 4-\mathrm{C} 9-\mathrm{C} 8$ & $119.6(2)$ \\
\hline $\mathrm{O} 3-\mathrm{Gd} 1-\mathrm{O} 4^{\mathrm{i}}$ & $68.12(5)$ & $\mathrm{N} 2-\mathrm{C} 10-\mathrm{C} 11$ & $117.04(19)$ \\
\hline $\mathrm{O} 1-\mathrm{Gd} 1-\mathrm{N} 4$ & $72.72(6)$ & $\mathrm{N} 2-\mathrm{C} 10-\mathrm{H} 10 \mathrm{~A}$ & 108.0 \\
\hline $\mathrm{O} 2-\mathrm{Gd} 1-\mathrm{N} 4$ & $74.51(6)$ & $\mathrm{C} 11-\mathrm{C} 10-\mathrm{H} 10 \mathrm{~A}$ & 108.0 \\
\hline $\mathrm{O} 3-\mathrm{Gd} 1-\mathrm{N} 4$ & $145.21(6)$ & $\mathrm{N} 2-\mathrm{C} 10-\mathrm{H} 10 \mathrm{~B}$ & 108.0 \\
\hline $\mathrm{O} 4-\mathrm{Gd} 1-\mathrm{N} 4$ & $83.25(6)$ & $\mathrm{C} 11-\mathrm{C} 10-\mathrm{H} 10 \mathrm{~B}$ & 108.0 \\
\hline $\mathrm{O} 1-\mathrm{Gd} 1-\mathrm{N} 3$ & $137.96(6)$ & $\mathrm{H} 10 \mathrm{~A}-\mathrm{C} 10-\mathrm{H} 10 \mathrm{~B}$ & 107.3 \\
\hline $\mathrm{O} 2-\mathrm{Gd} 1-\mathrm{N} 3$ & $76.82(6)$ & $\mathrm{C} 12-\mathrm{C} 11-\mathrm{C} 16$ & $119.5(2)$ \\
\hline $\mathrm{O} 3-\mathrm{Gd} 1-\mathrm{N} 3$ & $76.31(6)$ & $\mathrm{C} 12-\mathrm{C} 11-\mathrm{C} 10$ & $120.9(2)$ \\
\hline $\mathrm{O} 4 \mathrm{i}-\mathrm{Gd} 1-\mathrm{N} 3$ & $121.96(6)$ & $\mathrm{C} 16-\mathrm{C} 11-\mathrm{C} 10$ & $119.3(2)$ \\
\hline $\mathrm{N} 4-\mathrm{Gd} 1-\mathrm{N} 3$ & $137.77(6)$ & $\mathrm{C} 13-\mathrm{C} 12-\mathrm{C} 11$ & $121.6(3)$ \\
\hline $\mathrm{O} 1-\mathrm{Gd} 1-\mathrm{N} 2$ & $101.15(6)$ & $\mathrm{C} 13-\mathrm{C} 12-\mathrm{H} 12$ & 119.2 \\
\hline $\mathrm{O} 2-\mathrm{Gd} 1-\mathrm{N} 2$ & $76.69(6)$ & $\mathrm{C} 11-\mathrm{C} 12-\mathrm{H} 12$ & 119.2 \\
\hline $\mathrm{O} 3-\mathrm{Gd} 1-\mathrm{N} 2$ & $146.18(5)$ & $\mathrm{C} 14-\mathrm{C} 13-\mathrm{C} 12$ & 119.0 \\
\hline 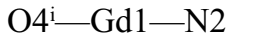 & $145.50(5)$ & $\mathrm{C} 14-\mathrm{C} 13-\mathrm{H} 13$ & 120.5 \\
\hline $\mathrm{N} 4-\mathrm{Gd} 1-\mathrm{N} 2$ & $65.63(6)$ & $\mathrm{C} 12-\mathrm{C} 13-\mathrm{H} 13$ & 120.5 \\
\hline $\mathrm{N} 3-\mathrm{Gd} 1-\mathrm{N} 2$ & $78.07(6)$ & $\mathrm{C} 13-\mathrm{C} 14-\mathrm{C} 15$ & $120.7(3)$ \\
\hline $\mathrm{O} 1-\mathrm{Gd} 1-\mathrm{N} 1$ & $74.44(6)$ & $\mathrm{C} 13-\mathrm{C} 14-\mathrm{H} 14$ & 119.6 \\
\hline $\mathrm{O} 2-\mathrm{Gd} 1-\mathrm{N} 1$ & $133.51(6)$ & $\mathrm{C} 15-\mathrm{C} 14-\mathrm{H} 14$ & 119.6 \\
\hline $\mathrm{O} 3-\mathrm{Gd} 1-\mathrm{N} 1$ & $80.10(5)$ & $\mathrm{C} 14-\mathrm{C} 15-\mathrm{C} 16$ & $121.1(3)$ \\
\hline $\mathrm{O} 4-\mathrm{Gd} 1-\mathrm{N} 1$ & $142.30(5)$ & $\mathrm{C} 14-\mathrm{C} 15-\mathrm{H} 15$ & 119.4 \\
\hline $\mathrm{N} 4-\mathrm{Gd} 1-\mathrm{N} 1$ & $116.30(6)$ & $\mathrm{C} 16-\mathrm{C} 15-\mathrm{H} 15$ & 119.4 \\
\hline $\mathrm{N} 3-\mathrm{Gd} 1-\mathrm{N} 1$ & $65.93(6)$ & $\mathrm{O} 2-\mathrm{C} 16-\mathrm{C} 15$ & $121.1(2)$ \\
\hline $\mathrm{N} 2-\mathrm{Gd} 1-\mathrm{N} 1$ & $69.43(6)$ & $\mathrm{O} 2-\mathrm{C} 16-\mathrm{C} 11$ & $120.8(2)$ \\
\hline $\mathrm{C} 17-\mathrm{N} 1-\mathrm{C} 1$ & $111.49(19)$ & $\mathrm{C} 15-\mathrm{C} 16-\mathrm{C} 11$ & $118.0(2)$ \\
\hline $\mathrm{C} 17-\mathrm{N} 1-\mathrm{C} 3$ & $105.29(18)$ & $\mathrm{N} 1-\mathrm{C} 17-\mathrm{C} 18$ & $115.3(2)$ \\
\hline $\mathrm{C} 1-\mathrm{N} 1-\mathrm{C} 3$ & $108.47(18)$ & $\mathrm{N} 1-\mathrm{C} 17-\mathrm{H} 17 \mathrm{~A}$ & 108.5 \\
\hline $\mathrm{C} 17-\mathrm{N} 1-\mathrm{Gd} 1$ & $108.34(13)$ & $\mathrm{C} 18-\mathrm{C} 17-\mathrm{H} 17 \mathrm{~A}$ & 108.5 \\
\hline $\mathrm{C} 1-\mathrm{N} 1-\mathrm{Gd} 1$ & $110.98(13)$ & $\mathrm{N} 1-\mathrm{C} 17-\mathrm{H} 17 \mathrm{~B}$ & 108.5 \\
\hline $\mathrm{C} 3-\mathrm{N} 1-\mathrm{Gd} 1$ & $112.16(13)$ & C18-C17-H17B & 108.5 \\
\hline $\mathrm{C} 23-\mathrm{N} 2-\mathrm{C} 2$ & $110.38(18)$ & $\mathrm{H} 17 \mathrm{~A}-\mathrm{C} 17-\mathrm{H} 17 \mathrm{~B}$ & 107.5 \\
\hline $\mathrm{C} 23-\mathrm{N} 2-\mathrm{C} 10$ & $110.90(18)$ & $\mathrm{N} 3-\mathrm{C} 18-\mathrm{C} 19$ & $121.8(3)$ \\
\hline $\mathrm{C} 2-\mathrm{N} 2-\mathrm{C} 10$ & $105.60(17)$ & $\mathrm{N} 3-\mathrm{C} 18-\mathrm{C} 17$ & $117.3(2)$ \\
\hline $\mathrm{C} 23-\mathrm{N} 2-\mathrm{Gd} 1$ & $107.83(13)$ & $\mathrm{C} 19-\mathrm{C} 18-\mathrm{C} 17$ & $120.8(2)$ \\
\hline $\mathrm{C} 2-\mathrm{N} 2-\mathrm{Gd} 1$ & $109.63(13)$ & $\mathrm{C} 20-\mathrm{C} 19-\mathrm{C} 18$ & $119.3(3)$ \\
\hline $\mathrm{C} 10-\mathrm{N} 2-\mathrm{Gd} 1$ & $112.50(13)$ & $\mathrm{C} 20-\mathrm{C} 19-\mathrm{H} 19$ & 120.4 \\
\hline $\mathrm{C} 22-\mathrm{N} 3-\mathrm{C} 18$ & $117.8(2)$ & $\mathrm{C} 18-\mathrm{C} 19-\mathrm{H} 19$ & 120.4 \\
\hline $\mathrm{C} 22-\mathrm{N} 3-\mathrm{Gd} 1$ & $123.69(17)$ & $\mathrm{C} 21-\mathrm{C} 20-\mathrm{C} 19$ & $119.4(3)$ \\
\hline $\mathrm{C} 18-\mathrm{N} 3-\mathrm{Gd} 1$ & $118.52(15)$ & $\mathrm{C} 21-\mathrm{C} 20-\mathrm{H} 20$ & 120.3 \\
\hline $\mathrm{C} 28-\mathrm{N} 4-\mathrm{C} 24$ & $118.3(2)$ & $\mathrm{C} 19-\mathrm{C} 20-\mathrm{H} 20$ & 120.3 \\
\hline $\mathrm{C} 28-\mathrm{N} 4-\mathrm{Gd} 1$ & $121.90(16)$ & $\mathrm{C} 20-\mathrm{C} 21-\mathrm{C} 22$ & $118.2(3)$ \\
\hline $\mathrm{C} 24-\mathrm{N} 4-\mathrm{Gd} 1$ & $119.79(15)$ & $\mathrm{C} 20-\mathrm{C} 21-\mathrm{H} 21$ & 120.9 \\
\hline C9-O1-Gd1 & $135.00(15)$ & $\mathrm{C} 22-\mathrm{C} 21-\mathrm{H} 21$ & 120.9 \\
\hline $\mathrm{C} 16-\mathrm{O} 2-\mathrm{Gd} 1$ & $131.61(13)$ & $\mathrm{N} 3-\mathrm{C} 22-\mathrm{C} 21$ & $123.4(3)$ \\
\hline $\mathrm{C} 29-\mathrm{O} 3-\mathrm{Gd} 1$ & $119.02(13)$ & $\mathrm{N} 3-\mathrm{C} 22-\mathrm{H} 22$ & 118.3 \\
\hline $\mathrm{C} 29-\mathrm{O} 4-\mathrm{Gd} 1^{\mathrm{i}}$ & $119.12(13)$ & $\mathrm{C} 21-\mathrm{C} 22-\mathrm{H} 22$ & 118.3 \\
\hline $\mathrm{C} 30-\mathrm{O} 30-\mathrm{H} 30$ & 109.5 & $\mathrm{~N} 2-\mathrm{C} 23-\mathrm{C} 24$ & $113.32(18)$ \\
\hline
\end{tabular}




\begin{tabular}{|c|c|}
\hline $\mathrm{N} 1-\mathrm{C} 1-\mathrm{C} 2$ & $114.19(18)$ \\
\hline $\mathrm{N} 1-\mathrm{C} 1-\mathrm{H} 1 \mathrm{~A}$ & 108.7 \\
\hline $\mathrm{C} 2-\mathrm{C} 1-\mathrm{H} 1 \mathrm{~A}$ & 108.7 \\
\hline $\mathrm{N} 1-\mathrm{C} 1-\mathrm{H} 1 \mathrm{~B}$ & 108.7 \\
\hline $\mathrm{C} 2-\mathrm{C} 1-\mathrm{H} 1 \mathrm{~B}$ & 108.7 \\
\hline $\mathrm{H} 1 \mathrm{~A}-\mathrm{C} 1-\mathrm{H} 1 \mathrm{~B}$ & 107.6 \\
\hline $\mathrm{N} 2-\mathrm{C} 2-\mathrm{C} 1$ & $113.77(19)$ \\
\hline $\mathrm{N} 2-\mathrm{C} 2-\mathrm{H} 2 \mathrm{~A}$ & 108.8 \\
\hline $\mathrm{C} 1-\mathrm{C} 2-\mathrm{H} 2 \mathrm{~A}$ & 108.8 \\
\hline $\mathrm{N} 2-\mathrm{C} 2-\mathrm{H} 2 \mathrm{~B}$ & 108.8 \\
\hline $\mathrm{C} 1-\mathrm{C} 2-\mathrm{H} 2 \mathrm{~B}$ & 108.8 \\
\hline $\mathrm{H} 2 \mathrm{~A}-\mathrm{C} 2-\mathrm{H} 2 \mathrm{~B}$ & 107.7 \\
\hline $\mathrm{C} 4-\mathrm{C} 3-\mathrm{N} 1$ & $115.32(19)$ \\
\hline $\mathrm{C} 4-\mathrm{C} 3-\mathrm{H} 3 \mathrm{~A}$ & 108.4 \\
\hline $\mathrm{N} 1-\mathrm{C} 3-\mathrm{H} 3 \mathrm{~A}$ & 108.4 \\
\hline $\mathrm{C} 4-\mathrm{C} 3-\mathrm{H} 3 \mathrm{~B}$ & 108.4 \\
\hline $\mathrm{N} 1-\mathrm{C} 3-\mathrm{H} 3 \mathrm{~B}$ & 108.4 \\
\hline $\mathrm{H} 3 \mathrm{~A}-\mathrm{C} 3-\mathrm{H} 3 \mathrm{~B}$ & 107.5 \\
\hline $\mathrm{C} 9-\mathrm{C} 4-\mathrm{C} 5$ & $119.6(3)$ \\
\hline $\mathrm{C} 9-\mathrm{C} 4-\mathrm{C} 3$ & $119.0(2)$ \\
\hline $\mathrm{C} 5-\mathrm{C} 4-\mathrm{C} 3$ & $121.3(2)$ \\
\hline $\mathrm{C} 6-\mathrm{C} 5-\mathrm{C} 4$ & $120.6(3)$ \\
\hline $\mathrm{C} 6-\mathrm{C} 5-\mathrm{H} 5$ & 119.7 \\
\hline $\mathrm{C} 4-\mathrm{C} 5-\mathrm{H} 5$ & 119.7 \\
\hline $\mathrm{C} 7-\mathrm{C} 6-\mathrm{C} 5$ & $119.6(3)$ \\
\hline $\mathrm{C} 7-\mathrm{C} 6-\mathrm{H} 6$ & 120.2 \\
\hline $\mathrm{C} 5-\mathrm{C} 6-\mathrm{H} 6$ & 120.2 \\
\hline $\mathrm{C} 6-\mathrm{C} 7-\mathrm{C} 8$ & $121.1(3)$ \\
\hline $\mathrm{C} 6-\mathrm{C} 7-\mathrm{H} 7$ & 119.4 \\
\hline $\mathrm{C} 8-\mathrm{C} 7-\mathrm{H} 7$ & 119.4 \\
\hline $\mathrm{C} 7-\mathrm{C} 8-\mathrm{C} 9$ & $119.4(3)$ \\
\hline $\mathrm{C} 17-\mathrm{N} 1-\mathrm{C} 1-\mathrm{C} 2$ & $-85.0(2)$ \\
\hline $\mathrm{C} 3-\mathrm{N} 1-\mathrm{C} 1-\mathrm{C} 2$ & $159.5(2)$ \\
\hline $\mathrm{Gd} 1-\mathrm{N} 1-\mathrm{C} 1-\mathrm{C} 2$ & $35.9(2)$ \\
\hline $\mathrm{C} 23-\mathrm{N} 2-\mathrm{C} 2-\mathrm{C} 1$ & $-74.8(2)$ \\
\hline $\mathrm{C} 10-\mathrm{N} 2-\mathrm{C} 2-\mathrm{C} 1$ & $165.3(2)$ \\
\hline $\mathrm{Gd} 1-\mathrm{N} 2-\mathrm{C} 2-\mathrm{C} 1$ & $43.8(2)$ \\
\hline $\mathrm{N} 1-\mathrm{C} 1-\mathrm{C} 2-\mathrm{N} 2$ & $-56.1(3)$ \\
\hline $\mathrm{C} 17-\mathrm{N} 1-\mathrm{C} 3-\mathrm{C} 4$ & $-176.3(2)$ \\
\hline $\mathrm{C} 1-\mathrm{N} 1-\mathrm{C} 3-\mathrm{C} 4$ & $-56.9(2)$ \\
\hline $\mathrm{Gd} 1-\mathrm{N} 1-\mathrm{C} 3-\mathrm{C} 4$ & $66.1(2)$ \\
\hline $\mathrm{N} 1-\mathrm{C} 3-\mathrm{C} 4-\mathrm{C} 9$ & $-61.9(3)$ \\
\hline $\mathrm{N} 1-\mathrm{C} 3-\mathrm{C} 4-\mathrm{C} 5$ & $121.1(2)$ \\
\hline $\mathrm{C} 9-\mathrm{C} 4-\mathrm{C} 5-\mathrm{C} 6$ & $0.5(4)$ \\
\hline $\mathrm{C} 3-\mathrm{C} 4-\mathrm{C} 5-\mathrm{C} 6$ & $177.4(3)$ \\
\hline $\mathrm{C} 4-\mathrm{C} 5-\mathrm{C} 6-\mathrm{C} 7$ & $0.5(5)$ \\
\hline $\mathrm{C} 5-\mathrm{C} 6-\mathrm{C} 7-\mathrm{C} 8$ & $-0.6(5)$ \\
\hline
\end{tabular}

$\begin{array}{ll}\mathrm{N} 2-\mathrm{C} 23-\mathrm{H} 23 \mathrm{~A} & 108.9 \\ \mathrm{C} 24-\mathrm{C} 23-\mathrm{H} 23 \mathrm{~A} & 108.9 \\ \mathrm{~N} 2-\mathrm{C} 23-\mathrm{H} 23 \mathrm{~B} & 108.9 \\ \mathrm{C} 24-\mathrm{C} 23-\mathrm{H} 23 \mathrm{~B} & 108.9 \\ \mathrm{H} 23 \mathrm{~A}-\mathrm{C} 23-\mathrm{H} 23 \mathrm{~B} & 107.7 \\ \mathrm{~N} 4-\mathrm{C} 24-\mathrm{C} 25 & 122.1(2) \\ \mathrm{N} 4-\mathrm{C} 24-\mathrm{C} 23 & 116.5(2) \\ \mathrm{C} 25-\mathrm{C} 24-\mathrm{C} 23 & 121.4(2) \\ \mathrm{C} 26-\mathrm{C} 25-\mathrm{C} 24 & 119.0(3) \\ \mathrm{C} 26-\mathrm{C} 25-\mathrm{H} 25 & 120.5 \\ \mathrm{C} 24-\mathrm{C} 25-\mathrm{H} 25 & 120.5 \\ \mathrm{C} 25-\mathrm{C} 26-\mathrm{C} 27 & 119.5(3) \\ \mathrm{C} 25-\mathrm{C} 26-\mathrm{H} 26 & 120.2\end{array}$

$\mathrm{C} 25-\mathrm{C} 26-\mathrm{H} 26 \quad 120.2$

$\mathrm{C} 27-\mathrm{C} 26-\mathrm{H} 26 \quad 120.2$

$\mathrm{C} 26-\mathrm{C} 27-\mathrm{C} 28$

$\mathrm{C} 26-\mathrm{C} 27-\mathrm{H} 27 \quad 120.9$

$\mathrm{C} 28-\mathrm{C} 27-\mathrm{H} 27 \quad 120.9$

$\mathrm{N} 4-\mathrm{C} 28-\mathrm{C} 27 \quad 123.0(3)$

$\mathrm{N} 4-\mathrm{C} 28-\mathrm{H} 28$

$\mathrm{C} 27-\mathrm{C} 28-\mathrm{H} 28 \quad 118.5$

$\mathrm{O} 4-\mathrm{C} 29-\mathrm{O} 3 \quad 126.83$ (19)

$\mathrm{O} 4-\mathrm{C} 29-\mathrm{C} 29^{\mathrm{i}} \quad 116.6(2)$

$\mathrm{O} 3-\mathrm{C} 29-\mathrm{C} 29^{\mathrm{i}} \quad 116.6(2)$

$\mathrm{O} 30-\mathrm{C} 30-\mathrm{H} 30 \mathrm{~A} \quad 109.5$

$\mathrm{O} 30-\mathrm{C} 30-\mathrm{H} 30 \mathrm{~B} \quad 109.5$

$\mathrm{H} 30 \mathrm{~A}-\mathrm{C} 30-\mathrm{H} 30 \mathrm{~B} \quad 109.5$

$\mathrm{O} 30-\mathrm{C} 30-\mathrm{H} 30 \mathrm{C} \quad 109.5$

$\mathrm{H} 30 \mathrm{~A}-\mathrm{C} 30-\mathrm{H} 30 \mathrm{C} \quad 109.5$

$\mathrm{H} 30 \mathrm{~B}-\mathrm{C} 30-\mathrm{H} 30 \mathrm{C} \quad 109.5$

$\mathrm{H} 1 \mathrm{~W}-\mathrm{O} 1 \mathrm{~W}-\mathrm{H} 2 \mathrm{~W}$

$\begin{array}{ll}\mathrm{C} 10-\mathrm{C} 11-\mathrm{C} 16-\mathrm{O} 2 & -7.4(3) \\ \mathrm{C} 12-\mathrm{C} 11-\mathrm{C} 16-\mathrm{C} 15 & -0.5(3) \\ \mathrm{C} 10-\mathrm{C} 11-\mathrm{C} 16-\mathrm{C} 15 & 173.5(2) \\ \mathrm{C} 1-\mathrm{N} 1-\mathrm{C} 17-\mathrm{C} 18 & 79.1(2) \\ \mathrm{C} 3-\mathrm{N} 1-\mathrm{C} 17-\mathrm{C} 18 & -163.5(2) \\ \mathrm{Gd} 1-\mathrm{N} 1-\mathrm{C} 17-\mathrm{C} 18 & -43.3(2) \\ \mathrm{C} 22-\mathrm{N} 3-\mathrm{C} 18-\mathrm{C} 19 & -1.7(4) \\ \mathrm{Gd} 1-\mathrm{N} 3-\mathrm{C} 18-\mathrm{C} 19 & 179.1(2) \\ \mathrm{C} 22-\mathrm{N} 3-\mathrm{C} 18-\mathrm{C} 17 & 174.2(2) \\ \mathrm{Gd} 1-\mathrm{N} 3-\mathrm{C} 18-\mathrm{C} 17 & -5.0(3) \\ \mathrm{N} 1-\mathrm{C} 17-\mathrm{C} 18-\mathrm{N} 3 & 34.3(3) \\ \mathrm{N} 1-\mathrm{C} 17-\mathrm{C} 18-\mathrm{C} 19 & -149.8(2) \\ \mathrm{N} 3-\mathrm{C} 18-\mathrm{C} 19-\mathrm{C} 20 & 3.0(4) \\ \mathrm{C} 17-\mathrm{C} 18-\mathrm{C} 19-\mathrm{C} 20 & -172.8(3) \\ \mathrm{C} 18-\mathrm{C} 19-\mathrm{C} 20-\mathrm{C} 21 & -1.6(5) \\ \mathrm{C} 19-\mathrm{C} 20-\mathrm{C} 21-\mathrm{C} 22 & -0.9(5)\end{array}$




$\begin{array}{llll}\mathrm{C} 6-\mathrm{C} 7-\mathrm{C} 8-\mathrm{C} 9 & -0.4(4) & \mathrm{C} 18-\mathrm{N} 3-\mathrm{C} 22-\mathrm{C} 21 & -1.0(4) \\ \mathrm{Gd} 1-\mathrm{O} 1-\mathrm{C} 9-\mathrm{C} 4 & 52.5(3) & \mathrm{Gd} 1-\mathrm{N} 3-\mathrm{C} 22-\mathrm{C} 21 & 178.2(2) \\ \mathrm{Gd} 1-\mathrm{O} 1-\mathrm{C} 9-\mathrm{C} 8 & -128.3(2) & \mathrm{C} 20-\mathrm{C} 21-\mathrm{C} 22-\mathrm{N} 3 & 2.3(4) \\ \mathrm{C} 5-\mathrm{C} 4-\mathrm{C} 9-\mathrm{O} 1 & 177.8(2) & \mathrm{C} 2-\mathrm{N} 2-\mathrm{C} 23-\mathrm{C} 24 & 165.50(19) \\ \mathrm{C} 3-\mathrm{C} 4-\mathrm{C} 9-\mathrm{O} 1 & 0.8(3) & \mathrm{C} 10-\mathrm{N} 2-\mathrm{C} 23-\mathrm{C} 24 & -77.8(2) \\ \mathrm{C} 5-\mathrm{C} 4-\mathrm{C} 9-\mathrm{C} 8 & -1.5(4) & \mathrm{Gd} 1-\mathrm{N} 2-\mathrm{C} 23-\mathrm{C} 24 & 45.8(2) \\ \mathrm{C} 3-\mathrm{C} 4-\mathrm{C} 9-\mathrm{C} 8 & -178.4(2) & \mathrm{C} 28-\mathrm{N} 4-\mathrm{C} 24-\mathrm{C} 25 & -1.2(4) \\ \mathrm{C} 7-\mathrm{C} 8-\mathrm{C} 9-\mathrm{O} 1 & -177.8(2) & \mathrm{Gd} 1-\mathrm{N} 4-\mathrm{C} 24-\mathrm{C} 25 & -178.42(18) \\ \mathrm{C} 7-\mathrm{C} 8-\mathrm{C} 9-\mathrm{C} 4 & 1.4(4) & \mathrm{C} 28-\mathrm{N} 4-\mathrm{C} 24-\mathrm{C} 23 & -179.2(2) \\ \mathrm{C} 23-\mathrm{N} 2-\mathrm{C} 10-\mathrm{C} 11 & 62.5(3) & \mathrm{Gd} 1-\mathrm{N} 4-\mathrm{C} 24-\mathrm{C} 23 & 3.6(3) \\ \mathrm{C} 2-\mathrm{N} 2-\mathrm{C} 10-\mathrm{C} 11 & -177.9(2) & \mathrm{N} 2-\mathrm{C} 23-\mathrm{C} 24-\mathrm{N} 4 & -35.2(3) \\ \mathrm{Gd} 1-\mathrm{N} 2-\mathrm{C} 10-\mathrm{C} 11 & -58.3(2) & \mathrm{N} 2-\mathrm{C} 23-\mathrm{C} 24-\mathrm{C} 25 & 146.8(2) \\ \mathrm{N} 2-\mathrm{C} 10-\mathrm{C} 11-\mathrm{C} 12 & -120.9(2) & \mathrm{N} 4-\mathrm{C} 24-\mathrm{C} 25-\mathrm{C} 26 & 1.1(4) \\ \mathrm{N} 2-\mathrm{C} 10-\mathrm{C} 11-\mathrm{C} 16 & 65.2(3) & \mathrm{C} 23-\mathrm{C} 24-\mathrm{C} 25-\mathrm{C} 26 & 179.1(3) \\ \mathrm{C} 16-\mathrm{C} 11-\mathrm{C} 12-\mathrm{C} 13 & 0.7(4) & \mathrm{C} 24-\mathrm{C} 25-\mathrm{C} 26-\mathrm{C} 27 & -0.3(4) \\ \mathrm{C} 10-\mathrm{C} 11-\mathrm{C} 12-\mathrm{C} 13 & -173.2(2) & \mathrm{C} 25-\mathrm{C} 26-\mathrm{C} 27-\mathrm{C} 28 & -0.4(5) \\ \mathrm{C} 11-\mathrm{C} 12-\mathrm{C} 13-\mathrm{C} 14 & 0.0(4) & \mathrm{C} 24-\mathrm{N} 4-\mathrm{C} 28-\mathrm{C} 27 & 0.5(4) \\ \mathrm{C} 12-\mathrm{C} 13-\mathrm{C} 14-\mathrm{C} 15 & -1.0(4) & \mathrm{Gd} 1-\mathrm{N} 4-\mathrm{C} 28-\mathrm{C} 27 & 177.6(2) \\ \mathrm{C} 13-\mathrm{C} 14-\mathrm{C} 15-\mathrm{C} 16 & 1.2(4) & \mathrm{C} 26-\mathrm{C} 27-\mathrm{C} 28-\mathrm{N} 4 & 0.3(5) \\ \mathrm{Gd} 1-\mathrm{O} 2-\mathrm{C} 16-\mathrm{C} 15 & 128.8(2) & \mathrm{Gd} 1-\mathrm{O} 4-\mathrm{C} 29-\mathrm{O} 3 & 174.05(18) \\ \mathrm{Gd} 1-\mathrm{O} 2-\mathrm{C} 16-\mathrm{C} 11 & -50.2(3) & \mathrm{Gd} 1-\mathrm{O} 4-\mathrm{C} 29-\mathrm{C} 29^{\mathrm{i}} & -5.8(3) \\ \mathrm{C} 14-\mathrm{C} 15-\mathrm{C} 16-\mathrm{O} 2 & -179.5(2) & \mathrm{Gd} 1-\mathrm{O} 3-\mathrm{C} 29-\mathrm{O} 4 & 174.16(17) \\ \mathrm{C} 14-\mathrm{C} 15-\mathrm{C} 16-\mathrm{C} 11 & -0.5(4) & \mathrm{Gd} 1-\mathrm{O} 3-\mathrm{C} 29-\mathrm{C} 29^{\mathrm{i}} & -6.0(3) \\ \mathrm{C} 12-\mathrm{C} 11-\mathrm{C} 16-\mathrm{O} 2 & 178.6(2) & & \end{array}$

Symmetry code: (i) $-x,-y+1,-z+1$.

Hydrogen-bond geometry $\left(\AA,{ }^{\circ}\right)$

\begin{tabular}{lllll}
\hline$D-\mathrm{H} \cdots A$ & $D-\mathrm{H}$ & $\mathrm{H} \cdots A$ & $D \cdots A$ & $D-\mathrm{H} \cdots A$ \\
\hline $\mathrm{O} 30-\mathrm{H} 30 \cdots \mathrm{O} 1$ & 0.84 & 1.80 & $2.643(3)$ & 177 \\
$\mathrm{C} 1-\mathrm{H} 1 B \cdots \mathrm{O} 1 W^{\mathrm{ii}}$ & 0.99 & 2.59 & $3.459(3)$ & 147 \\
$\mathrm{O} 1 W-\mathrm{H} 1 W^{\cdots} \mathrm{O} 2^{\mathrm{i}}$ & $0.83(2)$ & $1.96(2)$ & $2.786(3)$ & $170(3)$ \\
$\mathrm{O} 1 W-\mathrm{H} 2 W \cdots \mathrm{O} 30$ & $0.88(2)$ & $1.87(2)$ & $2.745(3)$ & $171(4)$ \\
\hline
\end{tabular}

Symmetry codes: (i) $-x,-y+1,-z+1$; (ii) $-x+1,-y+1,-z+1$.

( $\mu$-Oxalato)bis $\left\{\left[N^{\prime}, N^{\prime}\right.\right.$-bis(2-oxidobenzyl- $\left.\kappa O\right)-N^{\prime}, N^{\prime}$-bis(pyridin-2-ylmethyl- $\kappa N$ )ethylenediamine-

$\left.\kappa^{2} N, N^{\prime}\right]$ dysprosium(III)\}-methanol-water (1/4/4) (2)

Crystal data

$\left[\mathrm{Dy}_{2}\left(\mathrm{C}_{28} \mathrm{H}_{28} \mathrm{~N}_{4} \mathrm{O}_{2}\right)_{2}\left(\mathrm{C}_{2} \mathrm{O}_{4}\right)\right] \cdot 4 \mathrm{CH}_{4} \mathrm{O} \cdot 4 \mathrm{H}_{2} \mathrm{O}$

$M_{r}=1518.34$

Triclinic, $P \overline{1}$

$a=9.883(2) \AA$

$b=12.838(3) \AA$

$c=14.832(4) \AA$

$\alpha=68.213(9)^{\circ}$

$\beta=74.653(8)^{\circ}$

$\gamma=70.552(8)^{\circ}$

$V=1626.3(7) \AA^{3}$

$$
\begin{aligned}
& Z=1 \\
& F(000)=768
\end{aligned}
$$

$D_{\mathrm{x}}=1.550 \mathrm{Mg} \mathrm{m}^{-3}$

Mo $K \alpha$ radiation, $\lambda=0.71073 \AA$

Cell parameters from 9284 reflections

$\theta=3.0-27.7^{\circ}$

$\mu=2.35 \mathrm{~mm}^{-1}$

$T=100 \mathrm{~K}$

Prism, colourless

$0.35 \times 0.16 \times 0.12 \mathrm{~mm}$ 


\section{Data collection}

Bruker D8 Venture/Photon 100 CMOS diffractometer

Radiation source: fine-focus sealed tube Graphite monochromator

Detector resolution: 10.4167 pixels $\mathrm{mm}^{-1}$ $\varphi$ and $\omega$ scans

Absorption correction: multi-scan (SADABS; Krause et al., 2015)

$T_{\text {min }}=0.629, T_{\max }=0.746$

Refinement

Refinement on $F^{2}$

Least-squares matrix: full

$R\left[F^{2}>2 \sigma\left(F^{2}\right)\right]=0.025$

$w R\left(F^{2}\right)=0.060$

$S=1.06$

7091 reflections

380 parameters

3 restraints

Primary atom site location: dual
93133 measured reflections

7091 independent reflections

6385 reflections with $I>2 \sigma(I)$

$R_{\text {int }}=0.059$

$\theta_{\max }=27.0^{\circ}, \theta_{\min }=3.0^{\circ}$

$h=-12 \rightarrow 12$

$k=-16 \rightarrow 16$

$l=-18 \rightarrow 18$
Secondary atom site location: difference Fourier map

Hydrogen site location: mixed

$\mathrm{H}$ atoms treated by a mixture of independent and constrained refinement

$w=1 /\left[\sigma^{2}\left(F_{\mathrm{o}}^{2}\right)+(0.0244 P)^{2}+1.9868 P\right]$ where $P=\left(F_{\mathrm{o}}{ }^{2}+2 F_{\mathrm{c}}{ }^{2}\right) / 3$

$(\Delta / \sigma)_{\max }=0.002$

$\Delta \rho_{\max }=1.94 \mathrm{e} \AA^{-3}$

$\Delta \rho_{\min }=-0.63$ e $\AA^{-3}$

\section{Special details}

Geometry. All esds (except the esd in the dihedral angle between two 1.s. planes) are estimated using the full covariance matrix. The cell esds are taken into account individually in the estimation of esds in distances, angles and torsion angles; correlations between esds in cell parameters are only used when they are defined by crystal symmetry. An approximate (isotropic) treatment of cell esds is used for estimating esds involving 1.s. planes.

Fractional atomic coordinates and isotropic or equivalent isotropic displacement parameters $\left(\hat{A}^{2}\right)$

\begin{tabular}{lllll}
\hline & $x$ & $y$ & $z$ & $U_{\text {iso }} * U_{\text {eq }}$ \\
\hline Dy1 & $0.85062(2)$ & $0.36072(2)$ & $0.69594(2)$ & $0.02699(5)$ \\
N1 & $0.6306(3)$ & $0.3122(2)$ & $0.66909(18)$ & $0.0352(5)$ \\
N2 & $0.7282(3)$ & $0.2394(2)$ & $0.86207(18)$ & $0.0351(5)$ \\
N3 & $0.9135(3)$ & $0.1612(2)$ & $0.66925(19)$ & $0.0383(6)$ \\
N4 & $0.8096(3)$ & $0.4397(2)$ & $0.83619(18)$ & $0.0370(6)$ \\
O1 & $0.6811(2)$ & $0.52962(19)$ & $0.66391(17)$ & $0.0430(5)$ \\
O2 & $1.0390(2)$ & $0.25545(19)$ & $0.77711(15)$ & $0.0370(5)$ \\
O3 & $0.8993(2)$ & $0.40098(19)$ & $0.52306(14)$ & $0.0370(5)$ \\
O4 & $0.9940(2)$ & $0.51292(19)$ & $0.38014(14)$ & $0.0362(5)$ \\
O30 & $0.7330(4)$ & $0.7243(3)$ & $0.5351(2)$ & $0.0764(9)$ \\
H30 & 0.718496 & 0.661941 & 0.576725 & $0.115^{*}$ \\
C1 & $0.5449(3)$ & $0.2587(3)$ & $0.7655(2)$ & $0.0439(8)$ \\
H1A & 0.472908 & 0.321693 & 0.789304 & $0.053^{*}$ \\
H1B & 0.490545 & 0.213183 & 0.754747 & $0.053^{*}$ \\
C2 & $0.6350(4)$ & $0.1817(3)$ & $0.8429(2)$ & $0.0420(7)$ \\
H2A & 0.698079 & 0.113165 & 0.823044 & $0.050^{*}$ \\
H2B & 0.570144 & 0.153596 & 0.904753 & $0.050^{*}$ \\
C3 & $0.5280(3)$ & $0.4178(3)$ & $0.6129(2)$ & $0.0425(7)$ \\
H3A & 0.583081 & 0.454137 & 0.548988 & $0.051^{*}$
\end{tabular}




\begin{tabular}{|c|c|c|c|c|}
\hline H3B & 0.453500 & 0.392579 & 0.598837 & $0.051^{*}$ \\
\hline $\mathrm{C} 4$ & $0.4534(3)$ & $0.5066(3)$ & $0.6633(2)$ & $0.0429(7)$ \\
\hline $\mathrm{C} 5$ & $0.3007(4)$ & 0.5435 (4) & $0.6832(3)$ & $0.0612(10)$ \\
\hline H5 & 0.244013 & 0.508835 & 0.666133 & $0.073 *$ \\
\hline C6 & $0.2325(5)$ & $0.6292(4)$ & $0.7271(4)$ & 0.0755 (14) \\
\hline H6 & 0.129331 & 0.653494 & 0.739876 & $0.091^{*}$ \\
\hline $\mathrm{C} 7$ & $0.3137(5)$ & 0.6797 (4) & $0.7526(3)$ & 0.0699 (13) \\
\hline $\mathrm{H} 7$ & 0.266094 & 0.738051 & 0.783716 & $0.084^{*}$ \\
\hline $\mathrm{C} 8$ & $0.4657(4)$ & $0.6458(3)$ & $0.7330(3)$ & $0.0541(9)$ \\
\hline H8 & 0.521300 & 0.680638 & 0.750940 & $0.065^{*}$ \\
\hline C9 & $0.5350(3)$ & $0.5603(3)$ & $0.6870(2)$ & $0.0414(7)$ \\
\hline $\mathrm{C} 10$ & $0.8356(4)$ & 0.1433 (3) & $0.9201(2)$ & $0.0403(7)$ \\
\hline $\mathrm{H} 10 \mathrm{~A}$ & 0.885352 & 0.087425 & 0.882625 & $0.048 *$ \\
\hline H10B & 0.781416 & 0.102000 & 0.982074 & $0.048 *$ \\
\hline C11 & $0.9506(3)$ & 0.1755 (3) & $0.9460(2)$ & $0.0367(6)$ \\
\hline $\mathrm{C} 12$ & $0.9649(4)$ & $0.1478(3)$ & $1.0434(2)$ & $0.0462(8)$ \\
\hline H12 & 0.894743 & 0.115714 & 1.094305 & $0.055^{*}$ \\
\hline $\mathrm{C} 13$ & $1.0791(4)$ & 0.1657 (3) & $1.0681(3)$ & $0.0538(9)$ \\
\hline H13 & 1.087727 & 0.145829 & 1.135118 & $0.065 *$ \\
\hline C14 & $1.1796(4)$ & $0.2126(3)$ & $0.9944(3)$ & $0.0552(9)$ \\
\hline H14 & 1.259234 & 0.224058 & 1.010739 & $0.066^{*}$ \\
\hline C15 & 1.1668 (4) & $0.2435(3)$ & 0.8968 (3) & $0.0458(8)$ \\
\hline H15 & 1.236347 & 0.277543 & 0.846914 & $0.055^{*}$ \\
\hline $\mathrm{C} 16$ & $1.0523(3)$ & $0.2253(3)$ & $0.8705(2)$ & $0.0354(6)$ \\
\hline $\mathrm{C} 17$ & $0.6850(4)$ & $0.2350(3)$ & $0.6072(2)$ & $0.0435(7)$ \\
\hline H17A & 0.706153 & 0.282658 & 0.537894 & $0.052 *$ \\
\hline H17B & 0.606877 & 0.200092 & 0.611962 & $0.052^{*}$ \\
\hline C18 & $0.8178(4)$ & $0.1396(3)$ & $0.6332(2)$ & $0.0423(7)$ \\
\hline C19 & $0.8446(5)$ & 0.0344 (3) & $0.6155(3)$ & $0.0616(10)$ \\
\hline H19 & 0.773484 & 0.019132 & 0.593305 & $0.074^{*}$ \\
\hline $\mathrm{C} 20$ & $0.9743(5)$ & $-0.0461(4)$ & $0.6304(3)$ & $0.0690(12)$ \\
\hline $\mathrm{H} 20$ & 0.995152 & -0.117133 & 0.616886 & $0.083^{*}$ \\
\hline $\mathrm{C} 21$ & $1.0750(5)$ & -0.0245 & $0.6651(3)$ & $0.0605(10)$ \\
\hline $\mathrm{H} 21$ & 1.166397 & -0.079264 & 0.675028 & $0.073^{*}$ \\
\hline $\mathrm{C} 22$ & $1.0391(4)$ & $0.0793(3)$ & $0.6851(3)$ & $0.0477(8)$ \\
\hline $\mathrm{H} 22$ & 1.106771 & 0.093468 & 0.711293 & $0.057 *$ \\
\hline C23 & $0.6396(3)$ & 0.3207 (3) & $0.9182(2)$ & $0.0432(7)$ \\
\hline $\mathrm{H} 23 \mathrm{~A}$ & 0.609733 & 0.275136 & 0.986865 & $0.052 *$ \\
\hline H23B & 0.550307 & 0.367032 & 0.889121 & $0.052^{*}$ \\
\hline $\mathrm{C} 24$ & $0.7191(3)$ & $0.4020(3)$ & $0.9188(2)$ & $0.0382(7)$ \\
\hline $\mathrm{C} 25$ & 0.6957 (4) & $0.4392(3)$ & $0.9992(3)$ & $0.0525(9)$ \\
\hline $\mathrm{H} 25$ & 0.629682 & 0.412743 & 1.056475 & $0.063 *$ \\
\hline $\mathrm{C} 26$ & $0.7693(5)$ & $0.5151(4)$ & $0.9953(3)$ & $0.0623(10)$ \\
\hline H26 & 0.756092 & 0.540626 & 1.050250 & $0.075^{*}$ \\
\hline $\mathrm{C} 27$ & $0.8620(5)$ & $0.5537(4)$ & $0.9110(3)$ & $0.0611(10)$ \\
\hline $\mathrm{H} 27$ & 0.912648 & 0.607246 & 0.906183 & $0.073^{*}$ \\
\hline $\mathrm{C} 28$ & $0.8803(4)$ & $0.5130(3)$ & $0.8334(3)$ & $0.0491(8)$ \\
\hline $\mathrm{H} 28$ & 0.945997 & 0.538471 & 0.775576 & $0.059 *$ \\
\hline
\end{tabular}




$\begin{array}{lllll}\text { C29 } & 0.9690(3) & 0.4748(3) & 0.4720(2) & 0.0312(6) \\ \text { C30 } & 0.8049(6) & 0.7736(4) & 0.5747(4) & 0.0874(16) \\ \text { H30A } & 0.794694 & 0.855829 & 0.536520 & 0.131^{*} \\ \text { H30B } & 0.908275 & 0.732038 & 0.571185 & 0.131^{*} \\ \text { H30C } & 0.761049 & 0.766725 & 0.643423 & 0.131^{*} \\ \text { O1W } & 0.6925(3) & 0.7827(4) & 0.3435(3) & 0.0742(9) \\ \text { H1W } & 0.768(3) & 0.763(4) & 0.307(3) & 0.090(17)^{*} \\ \text { H2W } & 0.711(6) & 0.746(4) & 0.4022(18) & 0.10(2)^{*}\end{array}$

Atomic displacement parameters $\left(\AA^{2}\right)$

\begin{tabular}{|c|c|c|c|c|c|c|}
\hline & $U^{11}$ & $U^{22}$ & $U^{33}$ & $U^{12}$ & $U^{13}$ & $U^{23}$ \\
\hline Dy1 & $0.02658(7)$ & $0.03170(7)$ & $0.02368(7)$ & $-0.01256(5)$ & $0.00064(4)$ & $-0.00858(5)$ \\
\hline N1 & $0.0363(13)$ & $0.0385(14)$ & $0.0313(12)$ & $-0.0163(11)$ & $-0.0087(10)$ & $-0.0036(10)$ \\
\hline N2 & $0.0336(12)$ & $0.0423(14)$ & $0.0304(12)$ & $-0.0180(11)$ & $-0.0002(10)$ & $-0.0087(11)$ \\
\hline N3 & $0.0425(14)$ & $0.0350(13)$ & 0.0359 (14) & $-0.0110(11)$ & -0.0056 & $-0.0093(11)$ \\
\hline N4 & 0.0407 (14) & 0.0359 (13) & $0.0323(13)$ & $-0.0075(11)$ & $-0.0023(10)$ & $-0.0128(11)$ \\
\hline $\mathrm{O} 1$ & $0.0384(12)$ & $0.0367(12)$ & 0.0539 (14) & $-0.0058(9)$ & $-0.0119(10)$ & $-0.0147(10)$ \\
\hline $\mathrm{O} 2$ & $0.0304(10)$ & $0.0478(12)$ & $0.0321(11)$ & $-0.0086(9)$ & $-0.0052(8)$ & $-0.0131(9)$ \\
\hline $\mathrm{O} 3$ & $0.0450(12)$ & 0.0477 (12) & $0.0279(10)$ & $-0.0287(10)$ & $0.0019(9)$ & $-0.0130(9)$ \\
\hline $\mathrm{O} 4$ & $0.0443(11)$ & $0.0502(12)$ & $0.0235(10)$ & $-0.0289(10)$ & $0.0004(8)$ & $-0.0114(9)$ \\
\hline $\mathrm{O} 30$ & $0.101(2)$ & $0.0593(18)$ & $0.070(2)$ & $-0.0406(18)$ & -0.0105 (18) & $-0.0069(15)$ \\
\hline $\mathrm{C} 1$ & $0.0382(16)$ & $0.055(2)$ & $0.0406(17)$ & $-0.0265(15)$ & $-0.0046(13)$ & $-0.0054(15)$ \\
\hline $\mathrm{C} 2$ & 0.0447 (17) & 0.0437 (18) & $0.0367(16)$ & $-0.0230(15)$ & $-0.0010(13)$ & $-0.0054(14)$ \\
\hline $\mathrm{C} 3$ & 0.0405 (17) & 0.0515 (19) & $0.0359(16)$ & $-0.0180(15)$ & $-0.0163(13)$ & $-0.0018(14)$ \\
\hline $\mathrm{C} 4$ & $0.0380(16)$ & $0.0435(18)$ & $0.0363(17)$ & $-0.0063(14)$ & -0.0113 & $-0.0005(14)$ \\
\hline $\mathrm{C} 5$ & 0.0369 (18) & 0.064 & $0.065(3)$ & $-0.0081(18)$ & $-0.0109(17)$ & $-0.004(2)$ \\
\hline C6 & $0.041(2)$ & $0.069(3)$ & $0.079(3)$ & $0.005(2)$ & $0.000(2)$ & $-0.005(2)$ \\
\hline $\mathrm{C} 7$ & $0.065(3)$ & $0.051(2)$ & $0.054(2)$ & $0.014(2)$ & $0.002(2)$ & $-0.0050(19)$ \\
\hline $\mathrm{C} 8$ & $0.059(2)$ & 0.0417 (19) & $0.047(2)$ & $0.0006(17)$ & $-0.0085(17)$ & $-0.0100(16)$ \\
\hline C9 & $0.0391(16)$ & $0.0387(17)$ & $0.0331(16)$ & $-0.0033(13)$ & $-0.0080(13)$ & $-0.0013(13)$ \\
\hline $\mathrm{C} 10$ & $0.0472(18)$ & $0.0344(16)$ & $0.0356(16)$ & $-0.0159(14)$ & $-0.0071(13)$ & $-0.0021(13)$ \\
\hline $\mathrm{C} 11$ & $0.0384(16)$ & $0.0292(15)$ & $0.0391(16)$ & $-0.0056(12)$ & $-0.0068(13)$ & $-0.0093(12)$ \\
\hline $\mathrm{C} 12$ & $0.055(2)$ & $0.0376(17)$ & $0.0387(17)$ & $-0.0062(15)$ & $-0.0128(15)$ & $-0.0054(14)$ \\
\hline $\mathrm{C} 13$ & $0.067(2)$ & $0.052(2)$ & $0.046(2)$ & $-0.0057(18)$ & $-0.0245(18)$ & $-0.0162(17)$ \\
\hline $\mathrm{C} 14$ & $0.052(2)$ & $0.063(2)$ & $0.061(2)$ & $-0.0081(18)$ & $-0.0208(18)$ & $-0.029(2)$ \\
\hline C15 & $0.0351(16)$ & $0.059(2)$ & 0.0495 (19) & $-0.0113(15)$ & $-0.0069(14)$ & $-0.0251(17)$ \\
\hline C16 & $0.0334(15)$ & $0.0344(15)$ & $0.0368(16)$ & $-0.0017(12)$ & $-0.0071(12)$ & $-0.0147(13)$ \\
\hline $\mathrm{C} 17$ & 0.0513 (19) & $0.0443(18)$ & $0.0423(18)$ & $-0.0168(15)$ & $-0.0141(15)$ & $-0.0132(15)$ \\
\hline C18 & 0.0545 (19) & 0.0415 (17) & $0.0352(16)$ & $-0.0192(15)$ & $-0.0043(14)$ & $-0.0129(14)$ \\
\hline C19 & $0.083(3)$ & $0.051(2)$ & $0.064(2)$ & -0.019 & $-0.016(2)$ & $-0.0283(19)$ \\
\hline $\mathrm{C} 20$ & $0.096(3)$ & $0.048(2)$ & $0.069(3)$ & $-0.014(2)$ & $-0.011(2)$ & $-0.032(2)$ \\
\hline $\mathrm{C} 21$ & $0.069(3)$ & $0.042(2)$ & $0.056(2)$ & $0.0030(18)$ & -0.0085 (19) & $-0.0170(18)$ \\
\hline $\mathrm{C} 22$ & 0.0507 (19) & 0.0397 (18) & $0.048(2)$ & $-0.0061(15)$ & $-0.0073(15)$ & $-0.0143(15)$ \\
\hline $\mathrm{C} 23$ & $0.0352(16)$ & $0.054(2)$ & $0.0325(16)$ & $-0.0134(14)$ & $0.0043(12)$ & $-0.0098(14)$ \\
\hline $\mathrm{C} 24$ & $0.0368(15)$ & $0.0381(16)$ & $0.0290(15)$ & $0.0035(13)$ & $-0.0053(12)$ & $-0.0105(13)$ \\
\hline $\mathrm{C} 25$ & $0.054(2)$ & $0.061(2)$ & 0.0399 (19) & $-0.0074(18)$ & $-0.0015(15)$ & $-0.0234(17)$ \\
\hline $\mathrm{C} 26$ & $0.075(3)$ & $0.071(3)$ & $0.048(2)$ & $-0.012(2)$ & -0.0042 (19) & $-0.036(2)$ \\
\hline
\end{tabular}




\begin{tabular}{lllllll}
$\mathrm{C} 27$ & $0.073(3)$ & $0.060(2)$ & $0.064(3)$ & $-0.023(2)$ & $-0.004(2)$ & $-0.035(2)$ \\
$\mathrm{C} 28$ & $0.061(2)$ & $0.0468(19)$ & $0.0449(19)$ & $-0.0197(17)$ & $-0.0011(16)$ & $-0.0204(16)$ \\
$\mathrm{C} 29$ & $0.0299(14)$ & $0.0376(15)$ & $0.0295(14)$ & $-0.0142(12)$ & $0.0009(11)$ & $-0.0134(12)$ \\
$\mathrm{C} 30$ & $0.111(4)$ & $0.073(3)$ & $0.093(4)$ & $-0.046(3)$ & $0.016(3)$ & $-0.044(3)$ \\
O1W & $0.0373(14)$ & $0.123(3)$ & $0.079(2)$ & $-0.0221(16)$ & $0.0020(15)$ & $-0.056(2)$ \\
\hline
\end{tabular}

Geometric parameters $\left(\hat{A},{ }^{\circ}\right)$

\begin{tabular}{|c|c|c|c|}
\hline Dy1-O1 & $2.230(2)$ & $\mathrm{C} 10-\mathrm{C} 11$ & $1.508(4)$ \\
\hline Dy1-O2 & $2.246(2)$ & $\mathrm{C} 10-\mathrm{H} 10 \mathrm{~A}$ & 0.9900 \\
\hline Dy1-O3 & $2.367(2)$ & $\mathrm{C} 10-\mathrm{H} 10 \mathrm{~B}$ & 0.9900 \\
\hline Dy $1-O 4^{i}$ & $2.3762(19)$ & $\mathrm{C} 11-\mathrm{C} 12$ & $1.388(5)$ \\
\hline Dy1-N4 & $2.523(3)$ & $\mathrm{C} 11-\mathrm{C} 16$ & $1.405(4)$ \\
\hline Dy $1-\mathrm{N} 3$ & $2.581(3)$ & $\mathrm{C} 12-\mathrm{C} 13$ & $1.384(5)$ \\
\hline Dy $1-\mathrm{N} 2$ & $2.606(2)$ & $\mathrm{C} 12-\mathrm{H} 12$ & 0.9500 \\
\hline Dy1-N1 & $2.612(2)$ & $\mathrm{C} 13-\mathrm{C} 14$ & $1.371(6)$ \\
\hline $\mathrm{N} 1-\mathrm{C} 17$ & $1.473(4)$ & C13-H13 & 0.9500 \\
\hline $\mathrm{N} 1-\mathrm{C} 1$ & $1.500(4)$ & $\mathrm{C} 14-\mathrm{C} 15$ & $1.381(5)$ \\
\hline $\mathrm{N} 1-\mathrm{C} 3$ & $1.501(4)$ & $\mathrm{C} 14-\mathrm{H} 14$ & 0.9500 \\
\hline $\mathrm{N} 2-\mathrm{C} 23$ & $1.483(4)$ & $\mathrm{C} 15-\mathrm{C} 16$ & $1.402(4)$ \\
\hline $\mathrm{N} 2-\mathrm{C} 10$ & $1.488(4)$ & $\mathrm{C} 15-\mathrm{H} 15$ & 0.9500 \\
\hline $\mathrm{N} 2-\mathrm{C} 2$ & $1.492(4)$ & $\mathrm{C} 17-\mathrm{C} 18$ & $1.490(5)$ \\
\hline $\mathrm{N} 3-\mathrm{C} 22$ & $1.343(4)$ & C17-H17A & 0.9900 \\
\hline N3-C18 & $1.345(4)$ & $\mathrm{C} 17-\mathrm{H} 17 \mathrm{~B}$ & 0.9900 \\
\hline $\mathrm{N} 4-\mathrm{C} 28$ & $1.330(4)$ & $\mathrm{C} 18-\mathrm{C} 19$ & $1.398(5)$ \\
\hline $\mathrm{N} 4-\mathrm{C} 24$ & $1.343(4)$ & $\mathrm{C} 19-\mathrm{C} 20$ & $1.363(6)$ \\
\hline $\mathrm{O} 1-\mathrm{C} 9$ & $1.348(4)$ & C19-H19 & 0.9500 \\
\hline $\mathrm{O} 2-\mathrm{C} 16$ & $1.324(4)$ & $\mathrm{C} 20-\mathrm{C} 21$ & $1.376(6)$ \\
\hline $\mathrm{O} 3-\mathrm{C} 29$ & $1.254(3)$ & $\mathrm{C} 20-\mathrm{H} 20$ & 0.9500 \\
\hline $\mathrm{O} 4-\mathrm{C} 29$ & $1.251(3)$ & $\mathrm{C} 21-\mathrm{C} 22$ & $1.384(5)$ \\
\hline $\mathrm{O} 30-\mathrm{C} 30$ & $1.431(6)$ & $\mathrm{C} 21-\mathrm{H} 21$ & 0.9500 \\
\hline $\mathrm{O} 30-\mathrm{H} 30$ & 0.8400 & $\mathrm{C} 22-\mathrm{H} 22$ & 0.9500 \\
\hline $\mathrm{C} 1-\mathrm{C} 2$ & $1.483(5)$ & $\mathrm{C} 23-\mathrm{C} 24$ & $1.506(5)$ \\
\hline $\mathrm{C} 1-\mathrm{H} 1 \mathrm{~A}$ & 0.9900 & $\mathrm{C} 23-\mathrm{H} 23 \mathrm{~A}$ & 0.9900 \\
\hline $\mathrm{C} 1-\mathrm{H} 1 \mathrm{~B}$ & 0.9900 & $\mathrm{C} 23-\mathrm{H} 23 \mathrm{~B}$ & 0.9900 \\
\hline $\mathrm{C} 2-\mathrm{H} 2 \mathrm{~A}$ & 0.9900 & $\mathrm{C} 24-\mathrm{C} 25$ & $1.381(5)$ \\
\hline $\mathrm{C} 2-\mathrm{H} 2 \mathrm{~B}$ & 0.9900 & $\mathrm{C} 25-\mathrm{C} 26$ & $1.374(6)$ \\
\hline $\mathrm{C} 3-\mathrm{C} 4$ & $1.479(5)$ & $\mathrm{C} 25-\mathrm{H} 25$ & 0.9500 \\
\hline $\mathrm{C} 3-\mathrm{H} 3 \mathrm{~A}$ & 0.9900 & $\mathrm{C} 26-\mathrm{C} 27$ & $1.373(6)$ \\
\hline C3-H3B & 0.9900 & $\mathrm{C} 26-\mathrm{H} 26$ & 0.9500 \\
\hline $\mathrm{C} 4-\mathrm{C} 9$ & $1.396(5)$ & $\mathrm{C} 27-\mathrm{C} 28$ & $1.379(5)$ \\
\hline $\mathrm{C} 4-\mathrm{C} 5$ & $1.407(5)$ & $\mathrm{C} 27-\mathrm{H} 27$ & 0.9500 \\
\hline $\mathrm{C} 5-\mathrm{C} 6$ & $1.376(7)$ & $\mathrm{C} 28-\mathrm{H} 28$ & 0.9500 \\
\hline $\mathrm{C} 5-\mathrm{H} 5$ & 0.9500 & $\mathrm{C} 29-\mathrm{C} 29^{\mathrm{i}}$ & $1.555(5)$ \\
\hline $\mathrm{C} 6-\mathrm{C} 7$ & $1.377(7)$ & $\mathrm{C} 30-\mathrm{H} 30 \mathrm{~A}$ & 0.9800 \\
\hline C6-H6 & 0.9500 & $\mathrm{C} 30-\mathrm{H} 30 \mathrm{~B}$ & 0.9800 \\
\hline $\mathrm{C} 7-\mathrm{C} 8$ & $1.400(6)$ & $\mathrm{C} 30-\mathrm{H} 30 \mathrm{C}$ & 0.9800 \\
\hline $\mathrm{C} 7-\mathrm{H} 7$ & 0.9500 & $\mathrm{O} 1 \mathrm{~W}-\mathrm{H} 1 \mathrm{~W}$ & $0.824(19)$ \\
\hline
\end{tabular}




\begin{tabular}{|c|c|c|c|}
\hline $\mathrm{C} 8-\mathrm{C} 9$ & $1.395(5)$ & $\mathrm{O} 1 \mathrm{~W}-\mathrm{H} 2 \mathrm{~W}$ & 0.859 (19) \\
\hline $\mathrm{C} 8-\mathrm{H} 8$ & 0.9500 & & \\
\hline $\mathrm{O} 1-\mathrm{Dy} 1-\mathrm{O} 2$ & $144.63(8)$ & $\mathrm{C} 9-\mathrm{C} 8-\mathrm{H} 8$ & 120.3 \\
\hline $\mathrm{O} 1-\mathrm{Dy} 1-\mathrm{O} 3$ & $84.42(8)$ & $\mathrm{C} 7-\mathrm{C} 8-\mathrm{H} 8$ & 120.3 \\
\hline $\mathrm{O} 2-\mathrm{Dy} 1-\mathrm{O} 3$ & $116.58(8)$ & $\mathrm{O} 1-\mathrm{C} 9-\mathrm{C} 8$ & $120.5(3)$ \\
\hline $\mathrm{O} 1-\mathrm{Dy} 1-\mathrm{O} 4^{\mathrm{i}}$ & $81.35(8)$ & $\mathrm{O} 1-\mathrm{C} 9-\mathrm{C} 4$ & $119.3(3)$ \\
\hline $\mathrm{O} 2-\mathrm{Dy} 1-\mathrm{O}^{\mathrm{i}}$ & $80.87(8)$ & $\mathrm{C} 8-\mathrm{C} 9-\mathrm{C} 4$ & $120.2(3)$ \\
\hline $\mathrm{O} 3-\mathrm{Dy} 1-\mathrm{O}^{\mathrm{i}}$ & $68.63(6)$ & $\mathrm{N} 2-\mathrm{C} 10-\mathrm{C} 11$ & $117.3(2)$ \\
\hline $\mathrm{O} 1-\mathrm{Dy} 1-\mathrm{N} 4$ & $72.89(8)$ & $\mathrm{N} 2-\mathrm{C} 10-\mathrm{H} 10 \mathrm{~A}$ & 108.0 \\
\hline $\mathrm{O} 2-\mathrm{Dy} 1-\mathrm{N} 4$ & $74.69(8)$ & $\mathrm{C} 11-\mathrm{C} 10-\mathrm{H} 10 \mathrm{~A}$ & 108.0 \\
\hline $\mathrm{O} 3-\mathrm{Dy} 1-\mathrm{N} 4$ & $145.79(8)$ & $\mathrm{N} 2-\mathrm{C} 10-\mathrm{H} 10 \mathrm{~B}$ & 108.0 \\
\hline $\mathrm{O} 4-\mathrm{Dy} 1-\mathrm{N} 4$ & $82.64(8)$ & $\mathrm{C} 11-\mathrm{C} 10-\mathrm{H} 10 \mathrm{~B}$ & 108.0 \\
\hline $\mathrm{O} 1-\mathrm{Dy} 1-\mathrm{N} 3$ & $138.31(8)$ & $\mathrm{H} 10 \mathrm{~A}-\mathrm{C} 10-\mathrm{H} 10 \mathrm{~B}$ & 107.2 \\
\hline $\mathrm{O} 2-\mathrm{Dy} 1-\mathrm{N} 3$ & $76.73(8)$ & $\mathrm{C} 12-\mathrm{C} 11-\mathrm{C} 16$ & $119.5(3)$ \\
\hline $\mathrm{O} 3-\mathrm{Dy} 1-\mathrm{N} 3$ & $75.05(8)$ & $\mathrm{C} 12-\mathrm{C} 11-\mathrm{C} 10$ & $120.9(3)$ \\
\hline 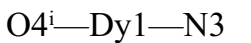 & $121.88(8)$ & $\mathrm{C} 16-\mathrm{C} 11-\mathrm{C} 10$ & $119.4(3)$ \\
\hline N4-Dy1-N3 & $138.23(8)$ & $\mathrm{C} 13-\mathrm{C} 12-\mathrm{C} 11$ & $121.5(3)$ \\
\hline $\mathrm{O} 1-\mathrm{Dy} 1-\mathrm{N} 2$ & $102.00(8)$ & $\mathrm{C} 13-\mathrm{C} 12-\mathrm{H} 12$ & 119.2 \\
\hline $\mathrm{O} 2-\mathrm{Dy} 1-\mathrm{N} 2$ & $77.22(8)$ & $\mathrm{C} 11-\mathrm{C} 12-\mathrm{H} 12$ & 119.2 \\
\hline O3-Dy1-N2 & $145.35(7)$ & $\mathrm{C} 14-\mathrm{C} 13-\mathrm{C} 12$ & $119.0(3)$ \\
\hline $\mathrm{O} 4-\mathrm{Dy} 1-\mathrm{N} 2$ & $145.73(7)$ & $\mathrm{C} 14-\mathrm{C} 13-\mathrm{H} 13$ & 120.5 \\
\hline N4-Dy1-N2 & $66.38(8)$ & $\mathrm{C} 12-\mathrm{C} 13-\mathrm{H} 13$ & 120.5 \\
\hline $\mathrm{N} 3-\mathrm{Dy} 1-\mathrm{N} 2$ & $78.08(8)$ & $\mathrm{C} 13-\mathrm{C} 14-\mathrm{C} 15$ & $120.9(3)$ \\
\hline O1-Dy1-N1 & $75.09(8)$ & $\mathrm{C} 13-\mathrm{C} 14-\mathrm{H} 14$ & 119.6 \\
\hline $\mathrm{O} 2-\mathrm{Dy} 1-\mathrm{N} 1$ & $133.82(8)$ & $\mathrm{C} 15-\mathrm{C} 14-\mathrm{H} 14$ & 119.6 \\
\hline $\mathrm{O} 3-\mathrm{Dy} 1-\mathrm{N} 1$ & $79.56(7)$ & $\mathrm{C} 14-\mathrm{C} 15-\mathrm{C} 16$ & $120.8(3)$ \\
\hline $\mathrm{O} 4-\mathrm{Dy} 1-\mathrm{N} 1$ & $141.93(7)$ & $\mathrm{C} 14-\mathrm{C} 15-\mathrm{H} 15$ & 119.6 \\
\hline N4-Dy1-N1 & $117.22(8)$ & $\mathrm{C} 16-\mathrm{C} 15-\mathrm{H} 15$ & 119.6 \\
\hline $\mathrm{N} 3-\mathrm{Dy} 1-\mathrm{N} 1$ & $65.83(8)$ & $\mathrm{O} 2-\mathrm{C} 16-\mathrm{C} 15$ & $121.0(3)$ \\
\hline $\mathrm{N} 2-\mathrm{Dy} 1-\mathrm{N} 1$ & $69.63(8)$ & $\mathrm{O} 2-\mathrm{C} 16-\mathrm{C} 11$ & $120.7(3)$ \\
\hline $\mathrm{C} 17-\mathrm{N} 1-\mathrm{C} 1$ & $111.6(3)$ & $\mathrm{C} 15-\mathrm{C} 16-\mathrm{C} 11$ & $118.3(3)$ \\
\hline $\mathrm{C} 17-\mathrm{N} 1-\mathrm{C} 3$ & $105.0(2)$ & $\mathrm{N} 1-\mathrm{C} 17-\mathrm{C} 18$ & $114.9(3)$ \\
\hline $\mathrm{C} 1-\mathrm{N} 1-\mathrm{C} 3$ & $107.6(2)$ & $\mathrm{N} 1-\mathrm{C} 17-\mathrm{H} 17 \mathrm{~A}$ & 108.5 \\
\hline C17-N1-Dy1 & $108.94(18)$ & $\mathrm{C} 18-\mathrm{C} 17-\mathrm{H} 17 \mathrm{~A}$ & 108.5 \\
\hline $\mathrm{C} 1-\mathrm{N} 1-\mathrm{Dy} 1$ & $111.06(17)$ & $\mathrm{N} 1-\mathrm{C} 17-\mathrm{H} 17 \mathrm{~B}$ & 108.5 \\
\hline C3-N1-Dy1 & $112.49(17)$ & $\mathrm{C} 18-\mathrm{C} 17-\mathrm{H} 17 \mathrm{~B}$ & 108.5 \\
\hline $\mathrm{C} 23-\mathrm{N} 2-\mathrm{C} 10$ & $110.9(2)$ & $\mathrm{H} 17 \mathrm{~A}-\mathrm{C} 17-\mathrm{H} 17 \mathrm{~B}$ & 107.5 \\
\hline $\mathrm{C} 23-\mathrm{N} 2-\mathrm{C} 2$ & $110.7(2)$ & N3-C18-C19 & $121.8(3)$ \\
\hline $\mathrm{C} 10-\mathrm{N} 2-\mathrm{C} 2$ & $105.4(2)$ & N3-C18-C17 & $117.4(3)$ \\
\hline $\mathrm{C} 23-\mathrm{N} 2-\mathrm{Dy} 1$ & $107.39(18)$ & $\mathrm{C} 19-\mathrm{C} 18-\mathrm{C} 17$ & $120.7(3)$ \\
\hline $\mathrm{C} 10-\mathrm{N} 2-\mathrm{Dy} 1$ & $112.67(17)$ & $\mathrm{C} 20-\mathrm{C} 19-\mathrm{C} 18$ & $119.1(4)$ \\
\hline $\mathrm{C} 2-\mathrm{N} 2-\mathrm{Dy} 1$ & $109.85(17)$ & $\mathrm{C} 20-\mathrm{C} 19-\mathrm{H} 19$ & 120.5 \\
\hline $\mathrm{C} 22-\mathrm{N} 3-\mathrm{C} 18$ & $117.8(3)$ & $\mathrm{C} 18-\mathrm{C} 19-\mathrm{H} 19$ & 120.5 \\
\hline C22-N3-Dy1 & $123.9(2)$ & $\mathrm{C} 19-\mathrm{C} 20-\mathrm{C} 21$ & $120.0(4)$ \\
\hline C18-N3-Dy1 & $118.3(2)$ & $\mathrm{C} 19-\mathrm{C} 20-\mathrm{H} 20$ & 120.0 \\
\hline $\mathrm{C} 28-\mathrm{N} 4-\mathrm{C} 24$ & $118.4(3)$ & $\mathrm{C} 21-\mathrm{C} 20-\mathrm{H} 20$ & 120.0 \\
\hline $\mathrm{C} 28-\mathrm{N} 4-\mathrm{Dy} 1$ & $122.3(2)$ & $\mathrm{C} 20-\mathrm{C} 21-\mathrm{C} 22$ & $118.0(4)$ \\
\hline
\end{tabular}




\begin{tabular}{|c|c|c|c|}
\hline C24-N4-Dy1 & $119.1(2)$ & $\mathrm{C} 20-\mathrm{C} 21-\mathrm{H} 21$ & 121.0 \\
\hline C9-O1-Dy1 & $133.9(2)$ & $\mathrm{C} 22-\mathrm{C} 21-\mathrm{H} 21$ & 121.0 \\
\hline $\mathrm{C} 16-\mathrm{O} 2-\mathrm{Dy} 1$ & $132.11(18)$ & $\mathrm{N} 3-\mathrm{C} 22-\mathrm{C} 21$ & $123.4(4)$ \\
\hline C29-O3-Dy1 & $118.66(17)$ & $\mathrm{N} 3-\mathrm{C} 22-\mathrm{H} 22$ & 118.3 \\
\hline $\mathrm{C} 29-\mathrm{O} 4-\mathrm{Dy} 1^{\mathrm{i}}$ & $119.02(17)$ & $\mathrm{C} 21-\mathrm{C} 22-\mathrm{H} 22$ & 118.3 \\
\hline $\mathrm{C} 30-\mathrm{O} 30-\mathrm{H} 30$ & 109.5 & $\mathrm{~N} 2-\mathrm{C} 23-\mathrm{C} 24$ & $113.1(2)$ \\
\hline $\mathrm{C} 2-\mathrm{C} 1-\mathrm{N} 1$ & $114.0(3)$ & $\mathrm{N} 2-\mathrm{C} 23-\mathrm{H} 23 \mathrm{~A}$ & 109.0 \\
\hline $\mathrm{C} 2-\mathrm{C} 1-\mathrm{H} 1 \mathrm{~A}$ & 108.8 & $\mathrm{C} 24-\mathrm{C} 23-\mathrm{H} 23 \mathrm{~A}$ & 109.0 \\
\hline $\mathrm{N} 1-\mathrm{C} 1-\mathrm{H} 1 \mathrm{~A}$ & 108.8 & $\mathrm{~N} 2-\mathrm{C} 23-\mathrm{H} 23 \mathrm{~B}$ & 109.0 \\
\hline $\mathrm{C} 2-\mathrm{C} 1-\mathrm{H} 1 \mathrm{~B}$ & 108.8 & $\mathrm{C} 24-\mathrm{C} 23-\mathrm{H} 23 \mathrm{~B}$ & 109.0 \\
\hline $\mathrm{N} 1-\mathrm{C} 1-\mathrm{H} 1 \mathrm{~B}$ & 108.8 & $\mathrm{H} 23 \mathrm{~A}-\mathrm{C} 23-\mathrm{H} 23 \mathrm{~B}$ & 107.8 \\
\hline $\mathrm{H} 1 \mathrm{~A}-\mathrm{C} 1-\mathrm{H} 1 \mathrm{~B}$ & 107.7 & $\mathrm{~N} 4-\mathrm{C} 24-\mathrm{C} 25$ & $121.9(3)$ \\
\hline $\mathrm{C} 1-\mathrm{C} 2-\mathrm{N} 2$ & $113.7(3)$ & $\mathrm{N} 4-\mathrm{C} 24-\mathrm{C} 23$ & $116.7(3)$ \\
\hline $\mathrm{C} 1-\mathrm{C} 2-\mathrm{H} 2 \mathrm{~A}$ & 108.8 & $\mathrm{C} 25-\mathrm{C} 24-\mathrm{C} 23$ & $121.4(3)$ \\
\hline $\mathrm{N} 2-\mathrm{C} 2-\mathrm{H} 2 \mathrm{~A}$ & 108.8 & $\mathrm{C} 26-\mathrm{C} 25-\mathrm{C} 24$ & $119.1(3)$ \\
\hline $\mathrm{C} 1-\mathrm{C} 2-\mathrm{H} 2 \mathrm{~B}$ & 108.8 & $\mathrm{C} 26-\mathrm{C} 25-\mathrm{H} 25$ & 120.5 \\
\hline $\mathrm{N} 2-\mathrm{C} 2-\mathrm{H} 2 \mathrm{~B}$ & 108.8 & $\mathrm{C} 24-\mathrm{C} 25-\mathrm{H} 25$ & 120.5 \\
\hline $\mathrm{H} 2 \mathrm{~A}-\mathrm{C} 2-\mathrm{H} 2 \mathrm{~B}$ & 107.7 & $\mathrm{C} 27-\mathrm{C} 26-\mathrm{C} 25$ & $119.2(3)$ \\
\hline $\mathrm{C} 4-\mathrm{C} 3-\mathrm{N} 1$ & $115.0(3)$ & $\mathrm{C} 27-\mathrm{C} 26-\mathrm{H} 26$ & 120.4 \\
\hline $\mathrm{C} 4-\mathrm{C} 3-\mathrm{H} 3 \mathrm{~A}$ & 108.5 & $\mathrm{C} 25-\mathrm{C} 26-\mathrm{H} 26$ & 120.4 \\
\hline $\mathrm{N} 1-\mathrm{C} 3-\mathrm{H} 3 \mathrm{~A}$ & 108.5 & $\mathrm{C} 26-\mathrm{C} 27-\mathrm{C} 28$ & $118.7(4)$ \\
\hline $\mathrm{C} 4-\mathrm{C} 3-\mathrm{H} 3 \mathrm{~B}$ & 108.5 & $\mathrm{C} 26-\mathrm{C} 27-\mathrm{H} 27$ & 120.7 \\
\hline $\mathrm{N} 1-\mathrm{C} 3-\mathrm{H} 3 \mathrm{~B}$ & 108.5 & $\mathrm{C} 28-\mathrm{C} 27-\mathrm{H} 27$ & 120.7 \\
\hline $\mathrm{H} 3 \mathrm{~A}-\mathrm{C} 3-\mathrm{H} 3 \mathrm{~B}$ & 107.5 & $\mathrm{~N} 4-\mathrm{C} 28-\mathrm{C} 27$ & $122.7(3)$ \\
\hline $\mathrm{C} 9-\mathrm{C} 4-\mathrm{C} 5$ & $118.8(3)$ & $\mathrm{N} 4-\mathrm{C} 28-\mathrm{H} 28$ & 118.7 \\
\hline $\mathrm{C} 9-\mathrm{C} 4-\mathrm{C} 3$ & $119.7(3)$ & $\mathrm{C} 27-\mathrm{C} 28-\mathrm{H} 28$ & 118.7 \\
\hline $\mathrm{C} 5-\mathrm{C} 4-\mathrm{C} 3$ & $121.4(3)$ & $\mathrm{O} 4-\mathrm{C} 29-\mathrm{O} 3$ & $126.9(3)$ \\
\hline $\mathrm{C} 6-\mathrm{C} 5-\mathrm{C} 4$ & $120.9(4)$ & $\mathrm{O} 4-\mathrm{C} 29-\mathrm{C} 29^{\mathrm{i}}$ & $116.1(3)$ \\
\hline $\mathrm{C} 6-\mathrm{C} 5-\mathrm{H} 5$ & 119.5 & $\mathrm{O} 3-\mathrm{C} 29-\mathrm{C} 29^{\mathrm{i}}$ & $117.0(3)$ \\
\hline $\mathrm{C} 4-\mathrm{C} 5-\mathrm{H} 5$ & 119.5 & $\mathrm{O} 30-\mathrm{C} 30-\mathrm{H} 30 \mathrm{~A}$ & 109.5 \\
\hline $\mathrm{C} 5-\mathrm{C} 6-\mathrm{C} 7$ & $120.0(4)$ & $\mathrm{O} 30-\mathrm{C} 30-\mathrm{H} 30 \mathrm{~B}$ & 109.5 \\
\hline $\mathrm{C} 5-\mathrm{C} 6-\mathrm{H} 6$ & 120.0 & $\mathrm{H} 30 \mathrm{~A}-\mathrm{C} 30-\mathrm{H} 30 \mathrm{~B}$ & 109.5 \\
\hline $\mathrm{C} 7-\mathrm{C} 6-\mathrm{H} 6$ & 120.0 & $\mathrm{O} 30-\mathrm{C} 30-\mathrm{H} 30 \mathrm{C}$ & 109.5 \\
\hline $\mathrm{C} 6-\mathrm{C} 7-\mathrm{C} 8$ & $120.5(4)$ & $\mathrm{H} 30 \mathrm{~A}-\mathrm{C} 30-\mathrm{H} 30 \mathrm{C}$ & 109.5 \\
\hline $\mathrm{C} 6-\mathrm{C} 7-\mathrm{H} 7$ & 119.7 & $\mathrm{H} 30 \mathrm{~B}-\mathrm{C} 30-\mathrm{H} 30 \mathrm{C}$ & 109.5 \\
\hline $\mathrm{C} 8-\mathrm{C} 7-\mathrm{H} 7$ & 119.7 & $\mathrm{H} 1 \mathrm{~W}-\mathrm{O} 1 \mathrm{~W}-\mathrm{H} 2 \mathrm{~W}$ & $105(4)$ \\
\hline $\mathrm{C} 9-\mathrm{C} 8-\mathrm{C} 7$ & $119.5(4)$ & & \\
\hline $\mathrm{C} 17-\mathrm{N} 1-\mathrm{C} 1-\mathrm{C} 2$ & $86.2(3)$ & $\mathrm{C} 10-\mathrm{C} 11-\mathrm{C} 16-\mathrm{O} 2$ & $7.2(4)$ \\
\hline $\mathrm{C} 3-\mathrm{N} 1-\mathrm{C} 1-\mathrm{C} 2$ & $-159.1(3)$ & $\mathrm{C} 12-\mathrm{C} 11-\mathrm{C} 16-\mathrm{C} 15$ & $1.0(4)$ \\
\hline Dy $1-\mathrm{N} 1-\mathrm{C} 1-\mathrm{C} 2$ & $-35.6(3)$ & $\mathrm{C} 10-\mathrm{C} 11-\mathrm{C} 16-\mathrm{C} 15$ & $-173.6(3)$ \\
\hline $\mathrm{N} 1-\mathrm{C} 1-\mathrm{C} 2-\mathrm{N} 2$ & $55.5(4)$ & $\mathrm{C} 1-\mathrm{N} 1-\mathrm{C} 17-\mathrm{C} 18$ & $-79.8(3)$ \\
\hline $\mathrm{C} 23-\mathrm{N} 2-\mathrm{C} 2-\mathrm{C} 1$ & $74.6(3)$ & $\mathrm{C} 3-\mathrm{N} 1-\mathrm{C} 17-\mathrm{C} 18$ & $163.9(3)$ \\
\hline $\mathrm{C} 10-\mathrm{N} 2-\mathrm{C} 2-\mathrm{C} 1$ & $-165.5(3)$ & Dy1-N1-C17-C18 & $43.2(3)$ \\
\hline $\mathrm{Dy} 1-\mathrm{N} 2-\mathrm{C} 2-\mathrm{C} 1$ & $-43.9(3)$ & $\mathrm{C} 22-\mathrm{N} 3-\mathrm{C} 18-\mathrm{C} 19$ & $2.1(5)$ \\
\hline $\mathrm{C} 17-\mathrm{N} 1-\mathrm{C} 3-\mathrm{C} 4$ & $177.2(3)$ & Dy1-N3-C18-C19 & $179.2(3)$ \\
\hline $\mathrm{C} 1-\mathrm{N} 1-\mathrm{C} 3-\mathrm{C} 4$ & $58.2(3)$ & $\mathrm{C} 22-\mathrm{N} 3-\mathrm{C} 18-\mathrm{C} 17$ & $-174.5(3)$ \\
\hline $\mathrm{Dy} 1-\mathrm{N} 1-\mathrm{C} 3-\mathrm{C} 4$ & $-64.4(3)$ & Dy $1-\mathrm{N} 3-\mathrm{C} 18-\mathrm{C} 17$ & $2.6(4)$ \\
\hline
\end{tabular}




\begin{tabular}{|c|c|c|c|}
\hline $\mathrm{N} 1-\mathrm{C} 3-\mathrm{C} 4-\mathrm{C} 9$ & $61.3(4)$ & $\mathrm{N} 1-\mathrm{C} 17-\mathrm{C} 18-\mathrm{N} 3$ & $-32.3(4)$ \\
\hline $\mathrm{N} 1-\mathrm{C} 3-\mathrm{C} 4-\mathrm{C} 5$ & $-122.4(3)$ & $\mathrm{N} 1-\mathrm{C} 17-\mathrm{C} 18-\mathrm{C} 19$ & $151.0(3)$ \\
\hline $\mathrm{C} 9-\mathrm{C} 4-\mathrm{C} 5-\mathrm{C} 6$ & $-1.4(5)$ & $\mathrm{N} 3-\mathrm{C} 18-\mathrm{C} 19-\mathrm{C} 20$ & $-3.4(6)$ \\
\hline $\mathrm{C} 3-\mathrm{C} 4-\mathrm{C} 5-\mathrm{C} 6$ & $-177.7(4)$ & $\mathrm{C} 17-\mathrm{C} 18-\mathrm{C} 19-\mathrm{C} 20$ & $173.1(4)$ \\
\hline $\mathrm{C} 4-\mathrm{C} 5-\mathrm{C} 6-\mathrm{C} 7$ & $-0.3(6)$ & $\mathrm{C} 18-\mathrm{C} 19-\mathrm{C} 20-\mathrm{C} 21$ & $1.8(7)$ \\
\hline $\mathrm{C} 5-\mathrm{C} 6-\mathrm{C} 7-\mathrm{C} 8$ & $0.9(7)$ & $\mathrm{C} 19-\mathrm{C} 20-\mathrm{C} 21-\mathrm{C} 22$ & $0.9(6)$ \\
\hline $\mathrm{C} 6-\mathrm{C} 7-\mathrm{C} 8-\mathrm{C} 9$ & $0.2(6)$ & $\mathrm{C} 18-\mathrm{N} 3-\mathrm{C} 22-\mathrm{C} 21$ & $0.8(5)$ \\
\hline Dy1-O1-C9-C8 & $126.2(3)$ & $\mathrm{Dy} 1-\mathrm{N} 3-\mathrm{C} 22-\mathrm{C} 21$ & $-176.1(3)$ \\
\hline Dy1-O1-C9-C4 & $-54.6(4)$ & $\mathrm{C} 20-\mathrm{C} 21-\mathrm{C} 22-\mathrm{N} 3$ & $-2.3(6)$ \\
\hline $\mathrm{C} 7-\mathrm{C} 8-\mathrm{C} 9-\mathrm{O} 1$ & $177.3(3)$ & $\mathrm{C} 10-\mathrm{N} 2-\mathrm{C} 23-\mathrm{C} 24$ & $77.3(3)$ \\
\hline $\mathrm{C} 7-\mathrm{C} 8-\mathrm{C} 9-\mathrm{C} 4$ & $-1.9(5)$ & $\mathrm{C} 2-\mathrm{N} 2-\mathrm{C} 23-\mathrm{C} 24$ & $-166.1(3)$ \\
\hline $\mathrm{C} 5-\mathrm{C} 4-\mathrm{C} 9-\mathrm{O} 1$ & $-176.8(3)$ & Dy $1-\mathrm{N} 2-\mathrm{C} 23-\mathrm{C} 24$ & $-46.2(3)$ \\
\hline $\mathrm{C} 3-\mathrm{C} 4-\mathrm{C} 9-\mathrm{O} 1$ & $-0.4(4)$ & $\mathrm{C} 28-\mathrm{N} 4-\mathrm{C} 24-\mathrm{C} 25$ & $1.4(5)$ \\
\hline $\mathrm{C} 5-\mathrm{C} 4-\mathrm{C} 9-\mathrm{C} 8$ & $2.4(5)$ & Dy $1-\mathrm{N} 4-\mathrm{C} 24-\mathrm{C} 25$ & $178.0(2)$ \\
\hline $\mathrm{C} 3-\mathrm{C} 4-\mathrm{C} 9-\mathrm{C} 8$ & $178.9(3)$ & $\mathrm{C} 28-\mathrm{N} 4-\mathrm{C} 24-\mathrm{C} 23$ & $179.0(3)$ \\
\hline $\mathrm{C} 23-\mathrm{N} 2-\mathrm{C} 10-\mathrm{C} 11$ & $-63.2(3)$ & Dy $1-\mathrm{N} 4-\mathrm{C} 24-\mathrm{C} 23$ & $-4.4(3)$ \\
\hline $\mathrm{C} 2-\mathrm{N} 2-\mathrm{C} 10-\mathrm{C} 11$ & $176.9(3)$ & $\mathrm{N} 2-\mathrm{C} 23-\mathrm{C} 24-\mathrm{N} 4$ & $36.1(4)$ \\
\hline $\mathrm{Dy} 1-\mathrm{N} 2-\mathrm{C} 10-\mathrm{C} 11$ & $57.1(3)$ & $\mathrm{N} 2-\mathrm{C} 23-\mathrm{C} 24-\mathrm{C} 25$ & $-146.3(3)$ \\
\hline $\mathrm{N} 2-\mathrm{C} 10-\mathrm{C} 11-\mathrm{C} 12$ & $121.6(3)$ & $\mathrm{N} 4-\mathrm{C} 24-\mathrm{C} 25-\mathrm{C} 26$ & $-1.3(5)$ \\
\hline $\mathrm{N} 2-\mathrm{C} 10-\mathrm{C} 11-\mathrm{C} 16$ & $-63.9(4)$ & $\mathrm{C} 23-\mathrm{C} 24-\mathrm{C} 25-\mathrm{C} 26$ & $-178.8(3)$ \\
\hline $\mathrm{C} 16-\mathrm{C} 11-\mathrm{C} 12-\mathrm{C} 13$ & $-1.4(5)$ & $\mathrm{C} 24-\mathrm{C} 25-\mathrm{C} 26-\mathrm{C} 27$ & $1.2(6)$ \\
\hline $\mathrm{C} 10-\mathrm{C} 11-\mathrm{C} 12-\mathrm{C} 13$ & $173.1(3)$ & $\mathrm{C} 25-\mathrm{C} 26-\mathrm{C} 27-\mathrm{C} 28$ & $-1.2(6)$ \\
\hline $\mathrm{C} 11-\mathrm{C} 12-\mathrm{C} 13-\mathrm{C} 14$ & $0.4(5)$ & $\mathrm{C} 24-\mathrm{N} 4-\mathrm{C} 28-\mathrm{C} 27$ & $-1.4(5)$ \\
\hline $\mathrm{C} 12-\mathrm{C} 13-\mathrm{C} 14-\mathrm{C} 15$ & $1.0(6)$ & Dy $1-\mathrm{N} 4-\mathrm{C} 28-\mathrm{C} 27$ & $-177.8(3)$ \\
\hline $\mathrm{C} 13-\mathrm{C} 14-\mathrm{C} 15-\mathrm{C} 16$ & $-1.4(6)$ & $\mathrm{C} 26-\mathrm{C} 27-\mathrm{C} 28-\mathrm{N} 4$ & $1.3(6)$ \\
\hline Dy1-O2-C16-C15 & $-129.4(3)$ & Dy 1 i-O4-C29-O3 & $-174.1(2)$ \\
\hline $\mathrm{Dy} 1-\mathrm{O} 2-\mathrm{C} 16-\mathrm{C} 11$ & $49.8(4)$ & Dy 1 i-O4-C29-C29i & $6.2(4)$ \\
\hline $\mathrm{C} 14-\mathrm{C} 15-\mathrm{C} 16-\mathrm{O} 2$ & $179.6(3)$ & Dy1-O3-C29-O4 & $-173.9(2)$ \\
\hline $\mathrm{C} 14-\mathrm{C} 15-\mathrm{C} 16-\mathrm{C} 11$ & $0.4(5)$ & Dy1-O3-C29-C29i & $5.8(4)$ \\
\hline $\mathrm{C} 12-\mathrm{C} 11-\mathrm{C} 16-\mathrm{O} 2$ & $-178.2(3)$ & & \\
\hline
\end{tabular}

Symmetry code: (i) $-x+2,-y+1,-z+1$.

Hydrogen-bond geometry $\left(A,{ }^{\circ}\right)$

\begin{tabular}{lllll}
\hline$D-\mathrm{H} \cdots A$ & $D-\mathrm{H}$ & $\mathrm{H} \cdots A$ & $D \cdots A$ & $D-\mathrm{H} \cdots A$ \\
\hline $\mathrm{O} 30-\mathrm{H} 30 \cdots \mathrm{O} 1$ & 0.84 & 1.80 & $2.636(4)$ & 178 \\
$\mathrm{C} 1-\mathrm{H} 1 B \cdots \mathrm{O} 1 W^{\mathrm{ii}}$ & 0.99 & 2.58 & $3.448(4)$ & 146 \\
$\mathrm{O} 1 W-\mathrm{H} 1 W \cdots \mathrm{O} 2^{\mathrm{i}}$ & $0.82(2)$ & $1.97(2)$ & $2.785(4)$ & $167(5)$ \\
$\mathrm{O} 1 W-\mathrm{H} 2 W \cdots \mathrm{O} 30$ & $0.86(2)$ & $1.95(3)$ & $2.759(5)$ & $158(5)$ \\
\hline
\end{tabular}

Symmetry codes: (i) $-x+2,-y+1,-z+1$; (ii) $-x+1,-y+1,-z+1$. 